\title{
Effects of High-speed Wind, Humidity, and Temperature on the Generation of a SARS-CoV-2 Aerosol; a Novel Point of View
}

Special Issue:

Special Issue on COVID-19 Aerosol Drivers, Impacts and Mitigation (XVI)

\section{Ata Nazari ${ }^{1}{ }^{*}$, Moharram Jafari ${ }^{1}$, Naser Rezaei ${ }^{1}$, Sevda Arash-Azad ${ }^{2}$, Faramarz Talati $^{1}$, Rahim Nejad-Rahim ${ }^{3}$, Farzad Taghizadeh-Hesary4 ${ }^{*}$, Farhad Taghizadeh-Hesary ${ }^{5}$}

\author{
${ }^{1}$ University of Tabriz, Department of Mechanical Engineering, Tabriz, Iran \\ ${ }^{2}$ Tabriz University of Medical Sciences, Department of Anesthesiology and Intensive Cares, \\ Tabriz, Iran \\ ${ }^{3}$ Urmia University of Medical Sciences, Department of Dermatology and Infectious Diseases, \\ Urmia, Iran \\ ${ }^{4}$ Shahid Beheshti University of Medical Sciences, Department of Clinical Oncology, Tehran, Iran \\ ${ }^{5}$ Social Science Research Institute, Tokai University, Hiratsuka-shi 259-1292, Kanagawa-ken, \\ Japan
}

\section{ABSTRACT}

The new coronavirus (SARS-CoV-2) is rapidly spreading across communities around the world. Respiratory droplet transmission is a common transmission route for many airborne diseases, including novel coronavirus disease (COVID-19). Wearing the face mask prevents respiratory droplet transmission. Both face mask leakage and non-use of the face mask under high-speed wind conditions can increase the risk of SARS-CoV-2 transmission. The respiratory droplets' behavior during sneezing or coughing (i.e., the size and the distance between droplets) depends on face mask wearing. The respiratory droplets during coughing and sneezing break apart into extremely small respiratory droplets (i.e., cloud of aerosol) upon interaction with the high-speed wind condition. The volume-of-fluid (VOF) method has been used to study the deformation and breakup of a single respiratory droplet placed in high-speed wind flow in the presence of smaller neighboring respiratory droplets. The effect of the diameter of respiratory droplets and the distance between them on the breakup mechanism has been investigated using open-source field operation and manipulation (OpenFOAM) libraries. The results are presented for the dimensionless distance and the normalized diameter of the secondary respiratory droplet in the ranges of 2 to 6 and 0.4 to 0.8 , respectively. According to the results of simulations, the spreading of SARS-CoV-2 increases with a decrease in respiratory droplet breakup time. In addition, the effects of ambient relative humidity and temperature on the cloud of respiratory particles were analyzed.

Keywords: COVID-19, SARS-CoV-2, Dynamic of respiratory droplets, VOF, OpenFOAM, Aerosol of viruses, Deformation and breakup, Ambient condition

\section{INTRODUCTION}

Since December 2019, novel coronavirus disease (COVID-19) has become a major concern for the global population. It has led to 915,393 deaths worldwide as of September 12, 2020 (Johns Hopkins University and Medicine, 2020). It simulated the clinical course of infection with two previously reported human coronaviruses-including severe acute respiratory syndrome coronavirus (SARS-CoV) and Middle East respiratory syndrome coronavirus (MERS-CoV) which was named severe acute respiratory syndrome coronavirus 2 (SARS-CoV-2) by the Coronavirus Study Group of the International Committee on Taxonomy of Viruses (Zhu et al., 2020). Various studies have 
reported that severe acute SARS-CoV-2 spreads mainly through respiratory droplets and aerosols (Yan et al., 2018; Asadi et al., 2019; Hsiao et al., 2020; National Academies of Sciences, 2020; Riediker and Morawska, 2020; Wilson et al., 2020). Respiratory droplets can transmit SARS-CoV-2 through the air (Xie et al., 2009; Rezaei et al., 2020; Nazari et al., 2021). These respiratory droplets can be exhaled during coughing, sneezing, or even talking (Xie et al., 2009). The World Health Organization (WHO) defined droplets as particles $\geq 5-10 \mu \mathrm{m}$ in diameter and aerosols as particles $<5 \mu \mathrm{m}$ (Wilson et al., 2020). Under high-speed wind conditions, respiratory droplets may convert to very small respiratory droplets in the wind direction. In this situation, the risk of SARS-CoV-2 transmission increases. Additionally, the deformation and breakup of respiratory droplets under wind conditions play a key role in the spread of new coronaviruses. Under high-speed wind conditions, the change in behavior of the respiratory droplets (i.e., their number and size) significantly increases the risk of SARS-CoV-2 transmission. Furthermore, based on the source of respiratory droplets and face mask wearing, different sizes of droplets (such as large ones and aerosols)-which behave differently in the outdoor environment-can be generated. Researchers have been seeking various solutions on how to control SARS-CoV-2 from spreading into communities (Ameri et al., 2020a, b; Bhardwaj and Agrawal, 2020; Li et al., 2020; Plog et al., 2020; Rakhsha et al., 2020; Siavashpour et al., 2020; Taghizadeh-Hesary and Akbari, 2020; Wang et al., 2020).

Dbouk and Drikakis (2020a) numerically studied airborne droplet transmission during coughing. They found that respiratory droplets could travel unexpected considerable distances depending on the high-speed wind conditions. Dbouk and Drikakis (2020b) demonstrated that respiratory droplets from coughing or sneezing traveled a distance less than $2 \mathrm{~m}$ in the case of zero-wind conditions. Bourouiba (2020) found that expelled respiratory droplets during human sneezing could travel up to $7-8 \mathrm{~m}$ at $36-108 \mathrm{~km} \mathrm{~h}^{-1}$ wind speeds. Chaudhuri et al. (2020) presented a model for the early phases of a COVID-19-like pandemic based on the aerodynamics and evaporation characteristics of respiratory droplets. They recognized that respiratory droplets exclusively contribute to the infection growth rate, which depends on the ambient temperature and relative humidity. Various researchers recommended the use of face masks in the public environment, and some of them believed that social distancing of $2 \mathrm{~m}$ may not be adequate during the COVID-19 outbreak (Schroter, 2020).

Dbouk and Drikakis (2020a) investigated the temporal behavior of respiratory droplet-droplet interactions on the high-speed wind. They found that respiratory droplet breakup occurs at different rates depending on environmental conditions, such as wind speed. In this work, the advanced stages of the breakup of the resulting respiratory particle clouds were observed, and a clear definition and quantification of the respiratory droplet breakup times were offered.

A comprehensive review of COVID-19 transmission via respiratory droplets was conducted by Carelli (2020), Chen (2020), Chen et al. (2020), and Drossinos and Stilianakis (2020). The infection probabilities of COVID-19 via large respiratory droplets were analyzed by Sun and Zhai (2020). They found 1.6-3.0 $\mathrm{m}$ as a safe social distance.

A better understanding of how respiratory droplets deform and break-up on high-speed wind may provide insight that contributes to mitigating SARS-CoV-2 spread. The influence of high-speed wind, relative humidity, and temperature on the respiratory droplets break-up (i.e., evaporation) is a complex topic. In this study, we introduce a novel numerical solution for heat and mass transfer for respiratory droplet interactions, which provide generated aerosol as a function of high-speed wind, relative humidity, and ambient temperature, as well as including virus fluid properties. To the best of our knowledge, the problem of deformation and interaction of multiple respiratory droplets in the high-speed wind flows has not been investigated in previous literature. The main aim of our work is to present predictions of the flow behavior and regimes resulting from the collision, deformation, and fragmentation of two and three respiratory droplets placed in a high-speed wind flow. This helps investigators gain a deep understanding of the behavior of complex wind flows, which are engaged with the dynamics of respiratory droplets. Then, different breakup regimes, including the stripping breakup regime, have been introduced. The simulations were carried out at a high-speed wind of $50 \mathrm{~km} \mathrm{~h}^{-1}$ with various configurations of the respiratory droplets, including different positions and diameters of the neighboring respiratory droplets.

The rest of the paper is arranged as follows. Section governing equation presents details of the mathematical modeling, the dimensionless numbers, and assumptions. The section Problem description and numerical technique discusses the details of the geometry, the description of the 
case studies, and the details of the numerical technique. After validation of our results using existing experimental and numerical data in the sections Validation of Results and Results and discussion presents a comprehensive discussion of the results and some advice. Finally, a conclusion is provided.

\section{GOVERNING EQUATION}

The relevant equations of motion are the momentum conservation law, the continuity equation, the energy equation, and the phase fraction equation in their incompressible form, respectively represented as:

$\rho \frac{D U}{D t}=-\nabla P+\int_{S(t)} \sigma \kappa\left(x^{\prime}\right) n\left(x^{\prime}\right) \delta\left(x-x^{\prime}\right) d S+\nabla \cdot \tau[0,1]$

$\nabla \cdot(\rho U)=0$

$\rho C \frac{D T}{D t}=\nabla \cdot(k \nabla T)+\frac{1}{2} \tau:\left(\nabla U+\nabla U^{T}\right)$

$\frac{\partial \rho_{k} \alpha_{k}}{\partial t}+\nabla \cdot\left(\rho_{k} U_{k} \alpha_{k}\right)=0$

where $p, \sigma, \tau$ are the pressure, the surface tension coefficient, and the deviatoric stress tensor, respectively. Stokes' hypothesis and the symmetry of the stress tensor are supposed to be valid. The surface force acting at the liquid-gas interface is considered by the surface integral in Eq. (1). $\kappa$ is twice the mean curvature of the interface, and $n$ is the unit normal vector of the interface. $\delta\left(x-x^{\prime}\right)$ denotes the Dirac-delta function, $x^{\prime} \in s(t)$ is a point on the interface and $x$ is the point at which the equation is evaluated (Tryggvason et al., 2001). In Eq. (3), $E=\rho e+1 / 2 \rho U^{2}$ is the total energy per unit volume, with $e$ being the internal energy per unit mass of the fluid and $\rho$ being the density.

Eq. (4) is provided by the volume-of-fluid (VOF) model, which governs the volume fraction $\alpha$ of one fluid in the mixture. The VOF method determines a phase fraction $(\alpha)$ for each cell within the computational domain. The cells having $\alpha$ equal to 1 and 0 contain only water and gas phases, respectively (Berberović et al., 2009).

In Eq. (3), the viscous dissipation term has been neglected. The effect of gravity is small for the corresponding size and lifetime of the respiratory droplets. For a given length scale $D$, three-time scales of advection, surface tension, and viscous effects exist. Based on these scales, the nondimensional Reynolds number and the Weber number can be obtained as follows.

$\operatorname{Re}=\frac{\rho_{g} u_{g} D}{\mu_{g}}, W e=\frac{\rho_{g} u_{g}{ }^{2} D}{\sigma}$

The characteristic parameters are the initial respiratory droplet diameter $D_{0}$, the gas velocity $u_{g}$, the inlet gas pressure $p_{g}$, the water density $\rho_{l}$, and the gas density at the inlet $\rho_{g}$.

$t^{*}=t \frac{u_{g}}{D_{0}} \sqrt{\frac{\rho_{g}}{\rho_{l}}}, \quad I^{*}=\frac{l}{D_{0}}, \quad d^{*}=\frac{d}{D_{0}}$,

where $t^{*}$ denotes the dimensionless time (Simpkins and Bales, 1972; Guildenbecher et al., 2009), $x^{*}, u^{*}$, and $a^{*}$ are the dimensionless position, velocity, and acceleration, respectively. $l^{*}$ and $d^{*}$ are the normalized distance and diameter of the secondary respiratory droplet, respectively.

The high-speed turbulent effects are modeled using the Reynolds-averaged form of Eqs. (1)(4) are called the Reynolds-averaged Navier-Stokes equations (or RANS equations) along with 
Table 1. Physical properties of water and air at the STP condition.

\begin{tabular}{llll}
\hline & $\rho\left(\mathrm{kg} \mathrm{m}^{-3}\right)$ & $\mu\left(\right.$ Pa.s $\left.\times 10^{-3}\right)$ & $\sigma\left(\mathrm{N} \mathrm{m}^{-1}\right)$ \\
\hline Air & 1.21 & 0.018 (Xie et al., 2007) & - \\
Water & 977 (Chaudhuri et al., 2020) & 1.1 & 0.0657 (Zhang et al., 2011) \\
\hline
\end{tabular}

the standard $k-\varepsilon$ turbulence model to address the closure problem. The initial values of the turbulent kinetic energy and the turbulent dissipation rate are $0.735 \mathrm{~m}^{2} \mathrm{~s}^{-2}$ and $3.835 \mathrm{~m}^{2} \mathrm{~S}^{-3}$, respectively (Smits and Dussauge, 2006; Rodrigues et al., 2016). For the complete description of the respiratory droplet-droplet collision, it is necessary to consider the physical properties involving viscosity, density, and surface tension coefficient at the standard condition. These parameters are listed in Table 1. It is well documented in the literature that average human respiratory droplets during sneezing and coughing consist of water, salt, proteins, and virus (SARS-CoV-2)/bacteria (Xie et al., 2007; Zhang et al., 2011; Chaudhuri et al., 2020).

\section{PROBLEM DESCRIPTION AND NUMERICAL TECHNIQUE}

Figs. 1 and 2 represent the schematic view of respiratory droplet ejection during a human cough or sneeze with or without wearing a face mask, respectively. The diameter and distance of the expelled respiratory droplets depend on the usage of the face mask. Given that larger and closer respiratory droplets can be expelled without wearing masks, the effects of the diameter and distance between the respiratory droplet on the breakup time, the catch-up time, and the collision morphology have been studied. Larger respiratory droplets may carry SARS-CoV-2 and thus constitute more danger in terms of COVID-19 transmission under high-speed wind conditions.

The open-source field operation and manipulation (OpenFOAM) CFD software package version 4.1 was used to perform the numerical simulations. The OpenFOAM codes were written in C++ programming language using the finite-volume numerical technique to solve the conservation of mass, energy, and momentum along with the equation of state in their Reynolds-averaged form. The base code of our solver is compressiblelnterFoam (Deshpande et al., 2012; OpenFOAM Foundation Ltd., 2016a, b). This solver can take into account different equations of state (EOS) models, but the stiffened-gas EOS is not included by default. Therefore, this feature is added to the basic codes by changing the source codes.

The second-order upwind scheme was used to handle the convective terms except for the phase-fraction term, which was discretized using the Van-Leer second-order scheme. The Gausslinear second-order approach was employed to address the diffusion terms. The PISO

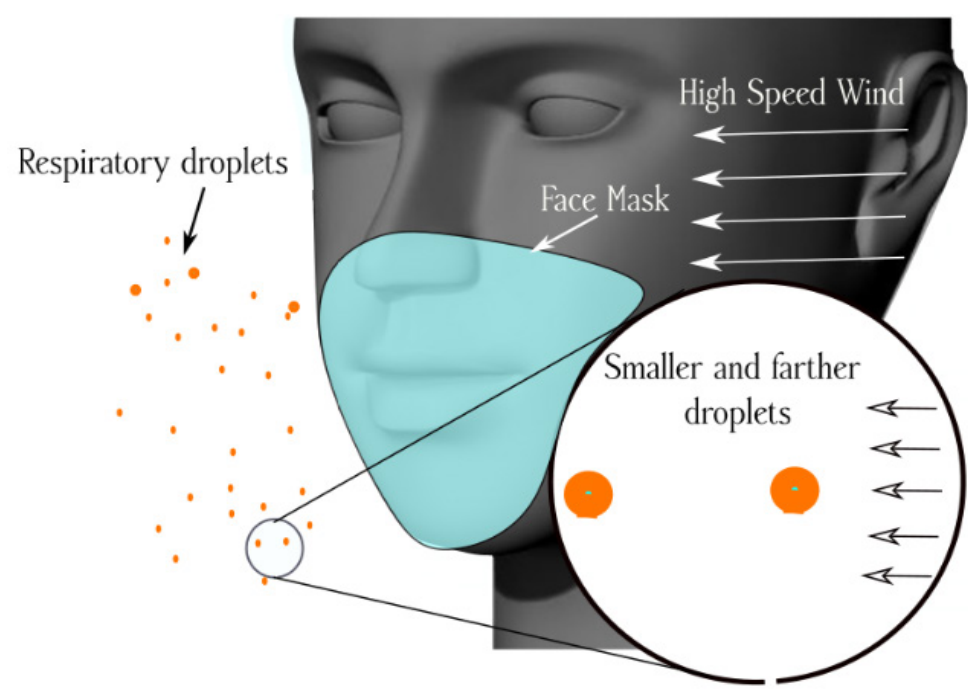

Fig. 1. Schematic view of respiratory droplet dispersion from a human cough or sneeze with a mask wearing at a high-speed wind of $50 \mathrm{~km} \mathrm{~h}^{-1}$. 


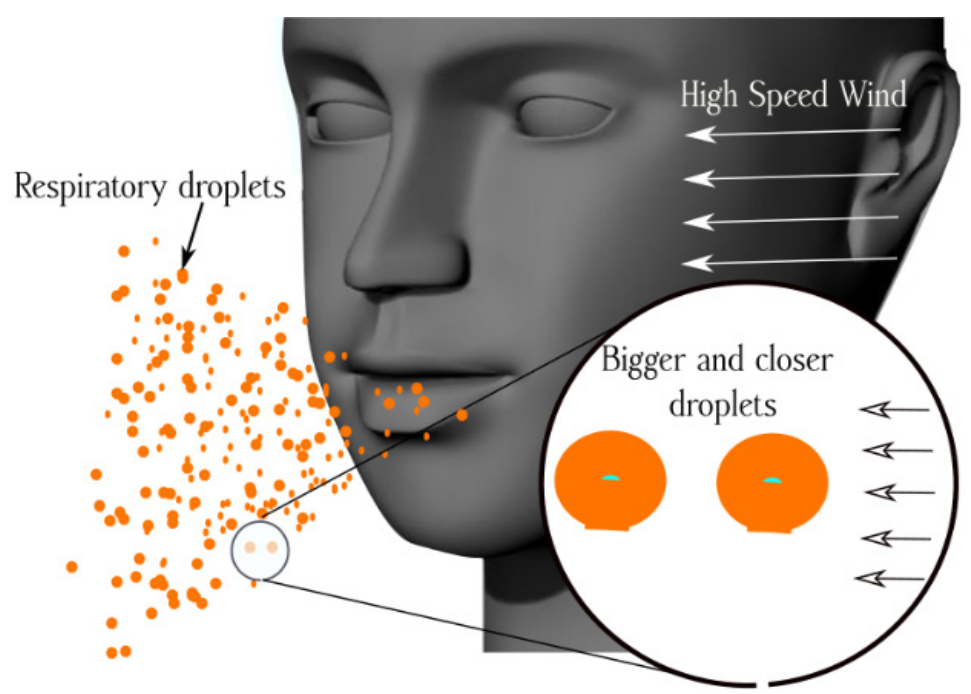

Fig. 2. Schematic view of respiratory droplet dispersion from a human cough or sneeze without mask wearing at a high-speed wind of $50 \mathrm{~km} \mathrm{~h}^{-1}$.

algorithm was applied to couple the pressure and the velocity. The under-relaxation factors for the pressure, momentum, and energy equations were $0.4,0.8$, and 0.9 , respectively. In addition, the minimum residuals for convergence of pressure, velocity, and temperature were $10^{-7}, 10^{-6}$, and $10^{-8}$, respectively.

Fig. 3 represents the schematic view of the computational domain, including the inlet and boundary conditions and the topology of the computational grid. To generate an effective computational mesh, we have used the block-mesh utility. The numerical mesh structure was gradually refined from the outer boundary with a cell size of $D_{0} / h=3$ towards the surface of the respiratory droplets $\left(D_{0} / h=24\right)$ by halving the size of the cells, in which $h$ is the cell size. The dimensions of the entire computational domain were $6 D_{0}$ and $22.5 D_{0}$ in the radial and streamwise directions, respectively. It is found that these sizes are large enough to guarantee that the results are not affected by the dimensions of the domain.

The fixed-value and fixed-flux pressure conditions are imposed at the inlet. The outlet-inlet is employed to model the outlet and far-field boundaries. The main respiratory droplet was supposed to be initially at rest and spherical with the diameter of $D_{0}=300 \mu \mathrm{m}$ placed at the distance $7.5 D_{0}$ from the inlet. The distance between the secondary nonidentical respiratory droplets and the main respiratory droplet in the tandem arrangement was $I^{*}=2-6$.

\section{VALIDATION OF RESULTS}

The comparison of our data with the experimental visualizations presented by Xie et al. (2007) for the two-dimensional numerical simulation of the morphology for the spherical water droplet is illustrated in Fig. 4. The airflow with a fixed magnitude moved over the respiratory droplet. The respiratory droplet temperature was set to $20^{\circ} \mathrm{C}$, and the ambient pressure, $p_{\infty}$, was $105 \mathrm{kPa}$. The drop reached the minimum pressure of $100 \mathrm{~Pa}$ at the leeward side. Fig. 4 demonstrates the comparison of the temporal droplet diameter between the present work and those of Xie et al. (2007). The maximum relative deviation of the droplet diameter for the two cases in Fig. 4 was approximately $3 \%$.

The grid independence test was carried out to compute the required number of numerical cells to obtain convergent results. To obtain the grid-independent results, simulations have been carried out on three different mesh topologies at the high-speed wind of $50 \mathrm{~km} \mathrm{~h}^{-1}$ past a single spherical respiratory droplet. Fig. 5 presents the VOF contours using the three mentioned numerical coarse, fine, and finest meshes. Three computational meshes were generated with the following details: $A=1500, B=2000$, and $C=2500$. Grids $B$ and $C$ produce almost identical results for the VOF contours with a relative error of less than $0.3 \%$. Hence, numerical grid $B$ chose to have a 


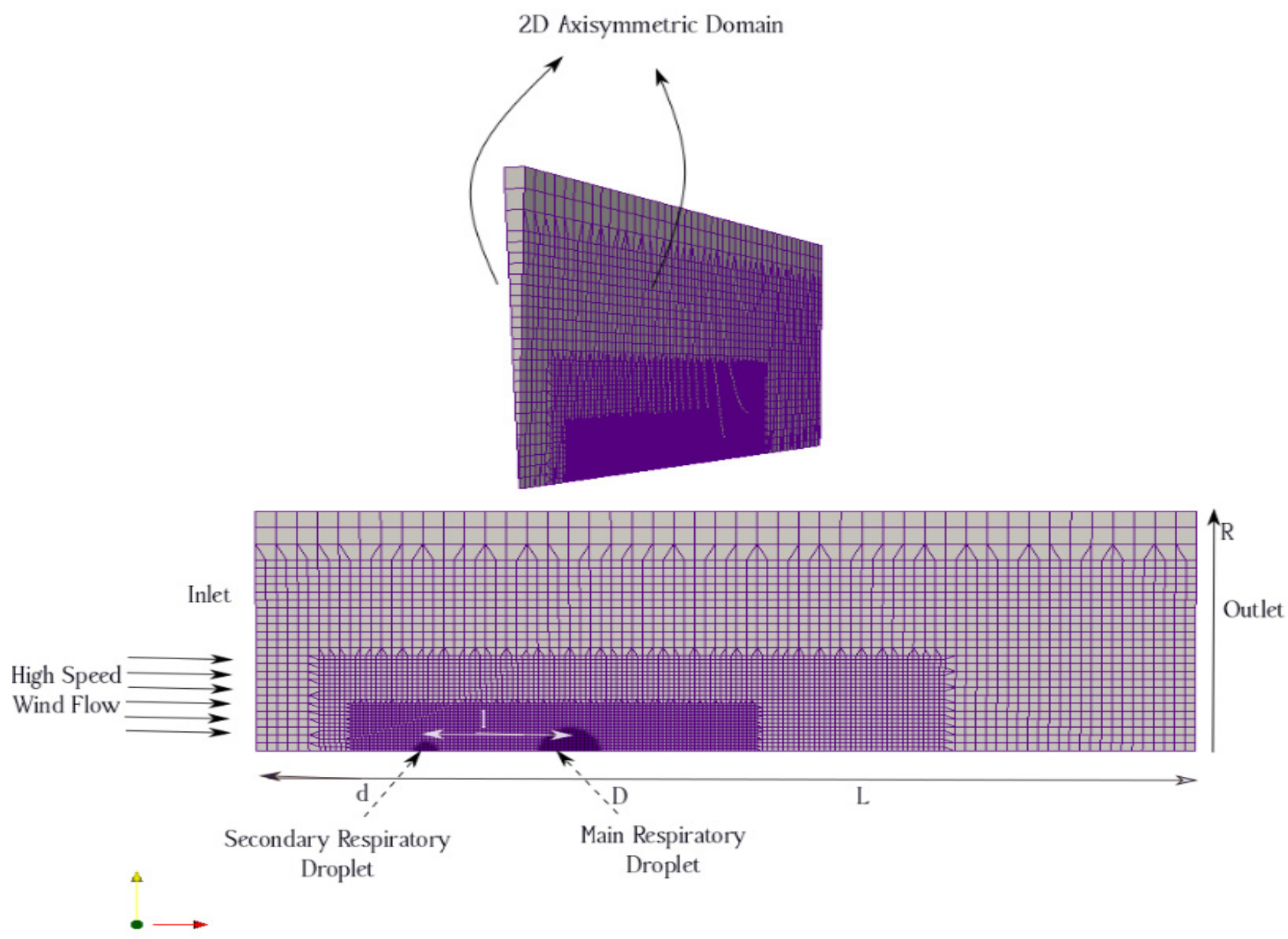

Fig. 3. Schematic two-dimensional (axisymmetric) view of the computational domain including the boundary conditions for the case of breakup of the respiratory droplet in high-speed wind flow.

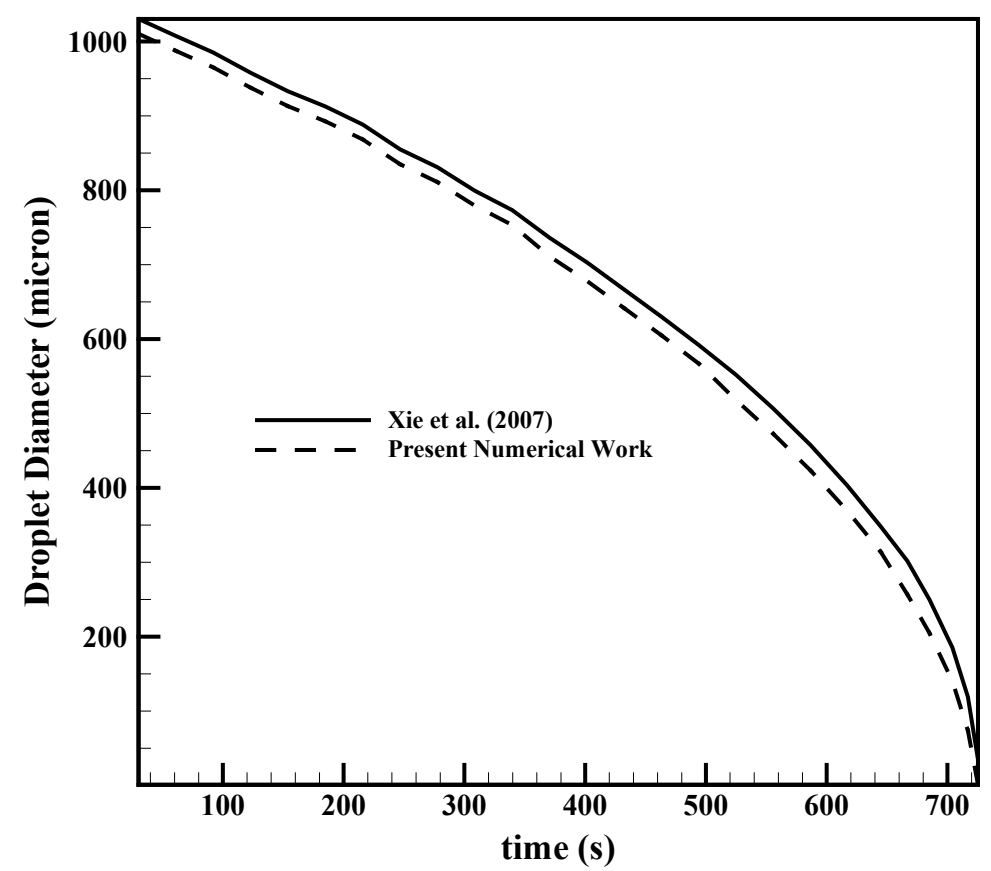

Fig. 4. Comparison of the temporal variation of the droplet diameter with the results of Xie et al. (2007). 


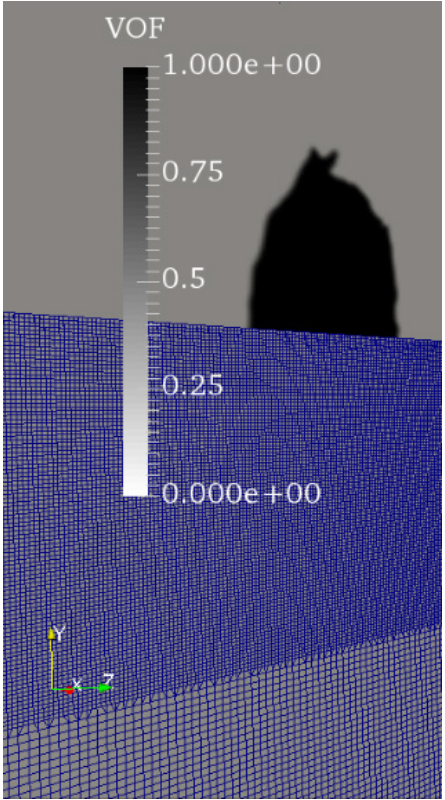

(a)

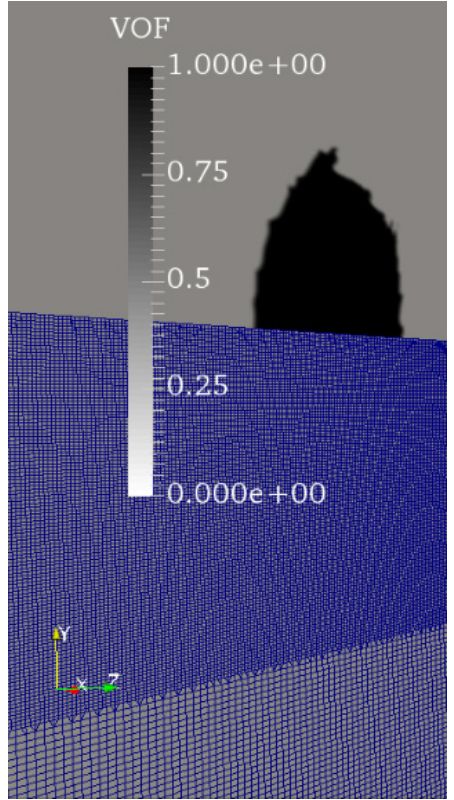

(b)

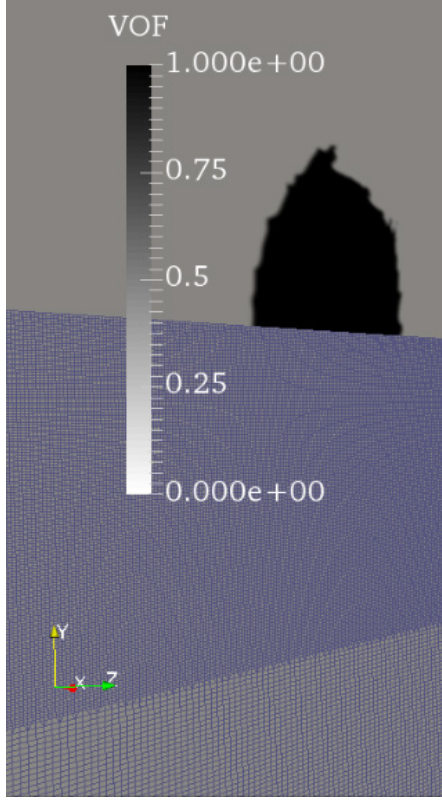

(c)

Fig. 5. The VOF contours around a single respiratory droplet obtained using (a) coarse mesh $A$, (b) fine mesh $B$, and (c) finest mesh $C$.

convergent solution with the optimized computational cost. A summary of the output of the grid independence test is shown in Fig. 5. The maximum skewness of the grid was 0.3 , which is quite suitable to obtain accurate results. The number of grid points over the smallest respiratory droplet in the finest mesh was 150 . The numerical predictions were also analyzed concerning the size of the time step. Therefore, three different time steps were considered, and the VOF contours were computed, yielding the results that prove that the time-step size-independent results have been obtained using the nondimensional time-step size of 0.02 . It should be noted that the time steps have been normalized by the inlet flow velocity and the main respiratory droplet diameter, $\left(\Delta t u_{g}\right) / D_{0}$.

\section{RESULTS AND DISCUSSION}

The present paper focuses on the morphology of a single respiratory droplet placed in a highspeed wind flow in the presence of small neighboring respiratory droplets. We assume that the secondary respiratory droplet can move with the high-speed wind flow and impact the main respiratory droplet. The dimensionless distance between two respiratory droplets was 2, 3, 4, 5, and 6 . The normalized diameters of the small respiratory droplets were $0.8,0.6$, and 0.4 . Due to the presence of other respiratory droplets inside the domain, the breakup process for the main respiratory droplet includes collision with the secondary respiratory droplet, penetration into the respiratory droplet, and splashing of the respiratory droplet. In addition, the effects of wind velocity, relative humidity, and ambient temperature have been studied. We defined the cloud of airborne to study the effect of the abovementioned parameters on the viruses, airborne, and very small respiratory droplets spreading during coughing and sneezing. Fig. 6 demonstrates the cloud of the created aerosol after the main respiratory droplet splashing. Three- and tworespiratory droplets before the collision and the cloud of the airborne and viruses after respiratory droplet splashing are shown schematically in Fig. 7.

\subsection{CASE A: INTERACTION OF TWO RESPIRATORY DROPLETS}

\subsubsection{Stage 1- Penetration}

Figs. 8 and $9\left(t^{*}=0.01-0.3\right)$ present the time-elapsed images of the two-dimensional respiratory droplet deformation. The normalized diameter of the first respiratory droplet in two cases is 0.4 


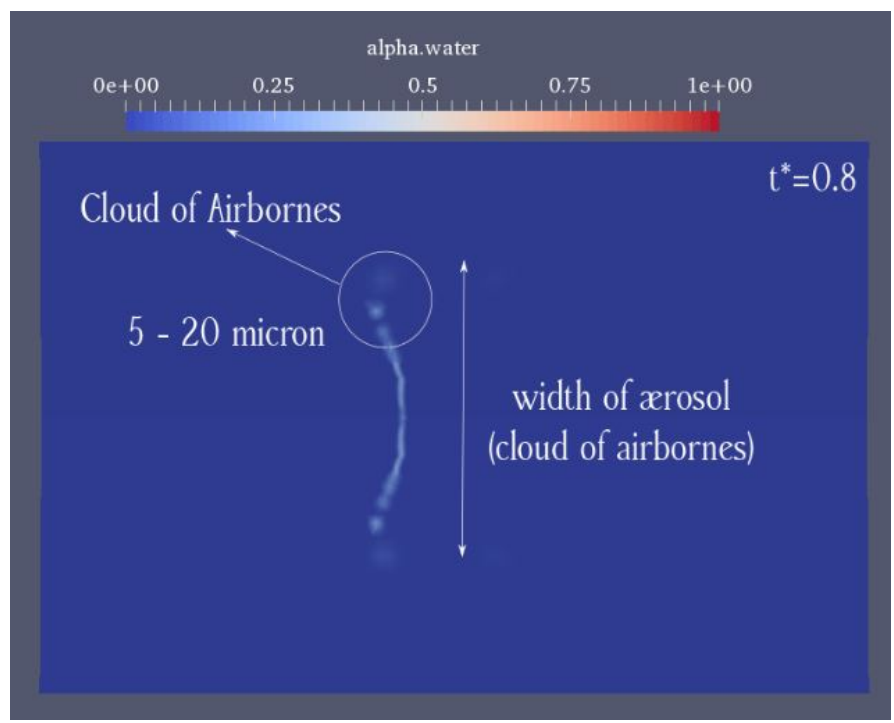

Fig. 6. View of the cloud of the airborne and the width of aerosol during the splashing of the main respiratory droplets.

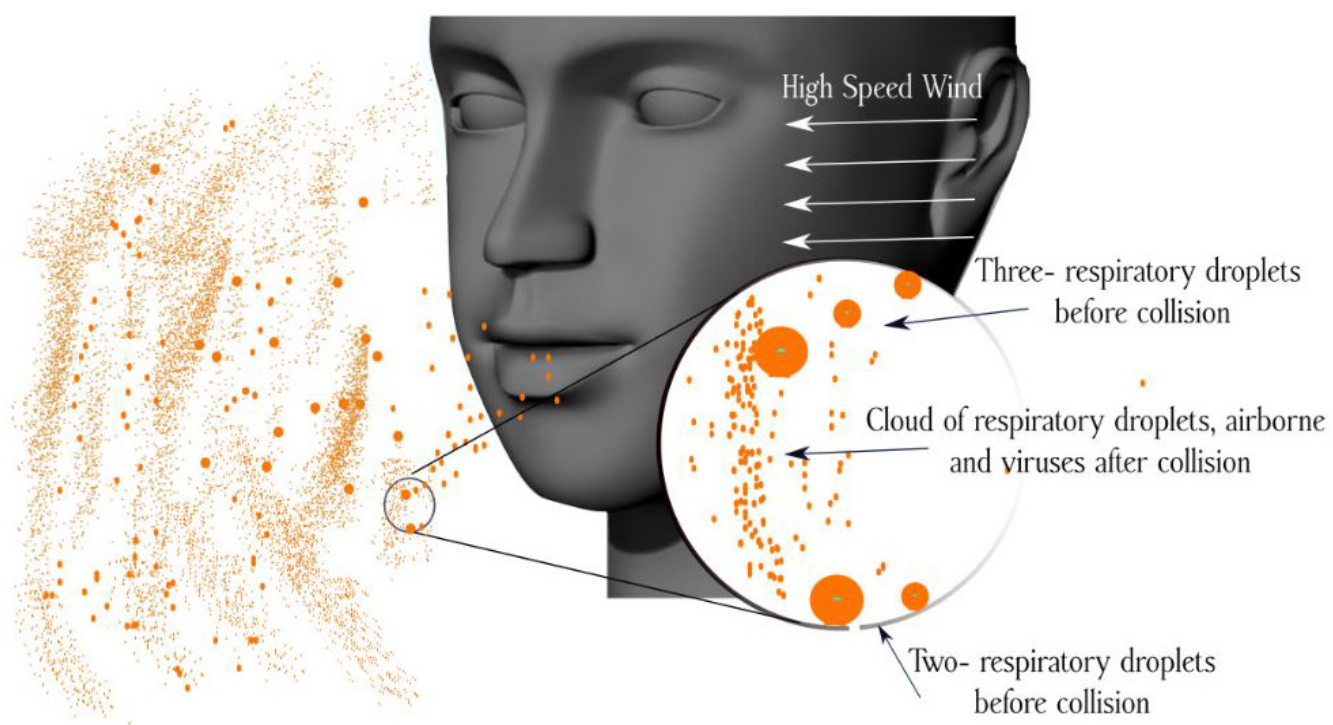

Fig. 7. Schematic view of the respiratory droplets cloud from a human cough or sneeze without mask wearing.

and 0.6, and the dimensionless distance between the two droplets is equal to 2, 3, 4, 5, and 6. At this stage, the smaller respiratory droplet moves with the velocity of the upstream flow and then strikes the main respiratory droplet. As the secondary respiratory droplet touches the main respiratory droplet, a liquid film starts to spread in the outward direction. A corona (in this case, is not the new coronavirus/SARS-CoV-2) around the secondary respiratory droplet is formed, which grows over time as the smaller respiratory droplet continues to feed the liquid film. Hence, the diameter of the liquid disk is greater in the case of the impact of the larger respiratory droplet. With continuous penetration of the smaller respiratory droplet into the main respiratory droplet, several coronas become visible on the frame, indicating that the timescale of the formation of the corona is much larger than the timescale of the deformation of the main respiratory droplet.

The comparison of the contours presented in Figs. 8 and 10 reveals that with increasing diameter of the secondary respiratory droplet, the time of penetration of the second respiratory droplet into the main respiratory droplet decreases. Clearly, when the larger respiratory droplet collides with the main respiratory droplet, the diameter of the created hole is larger in comparison 

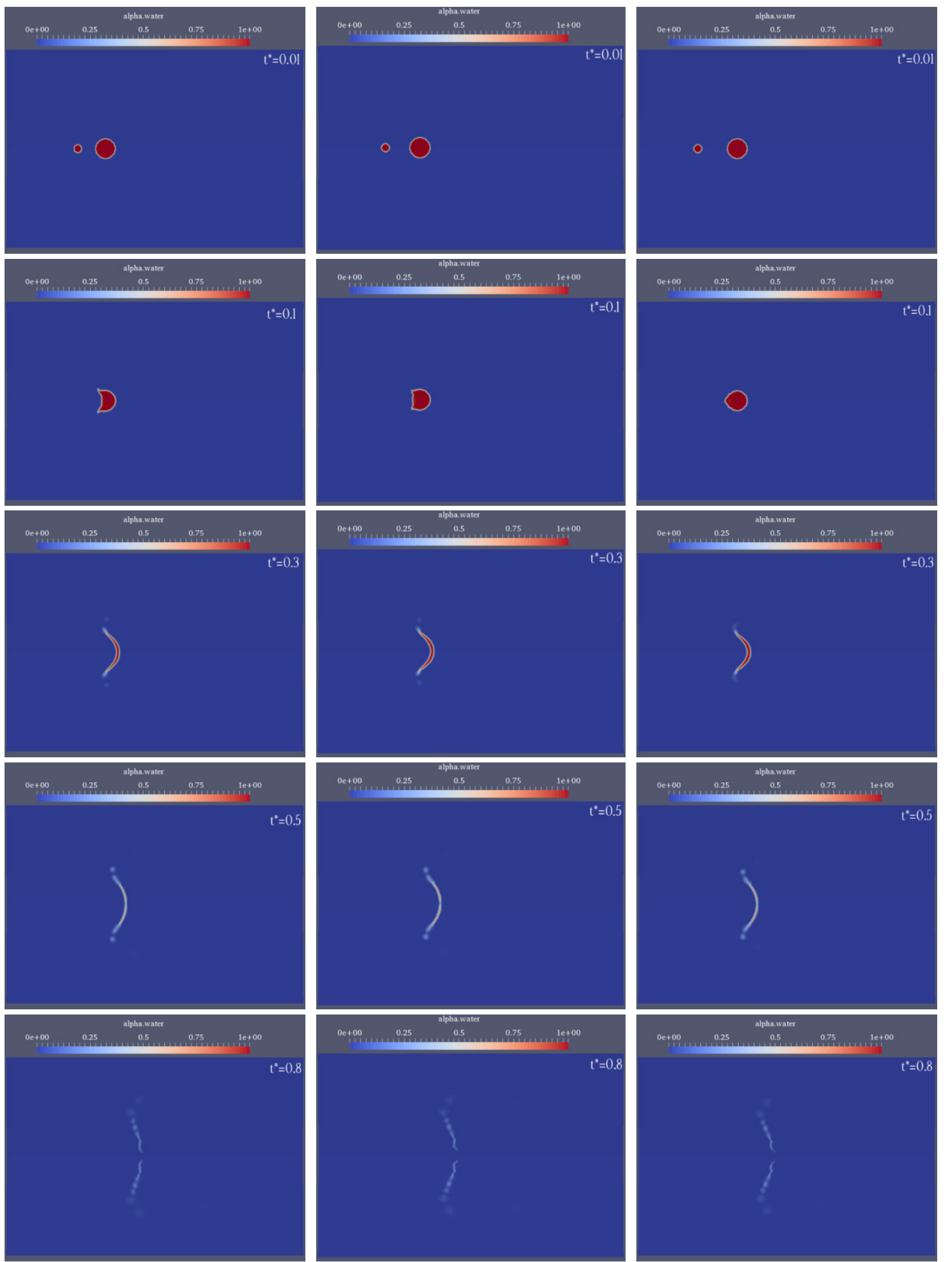

Fig. 8. Two-dimensional view of the two-respiratory droplet deformation at the penetrating and splashing stages. The dimensionless distance between the respiratory droplets is 2 (the first column), 3 (the second column), and 4 (the third column). The normalized diameter of the second respiratory droplet for the specified dimensionless distance is 0.4 . 

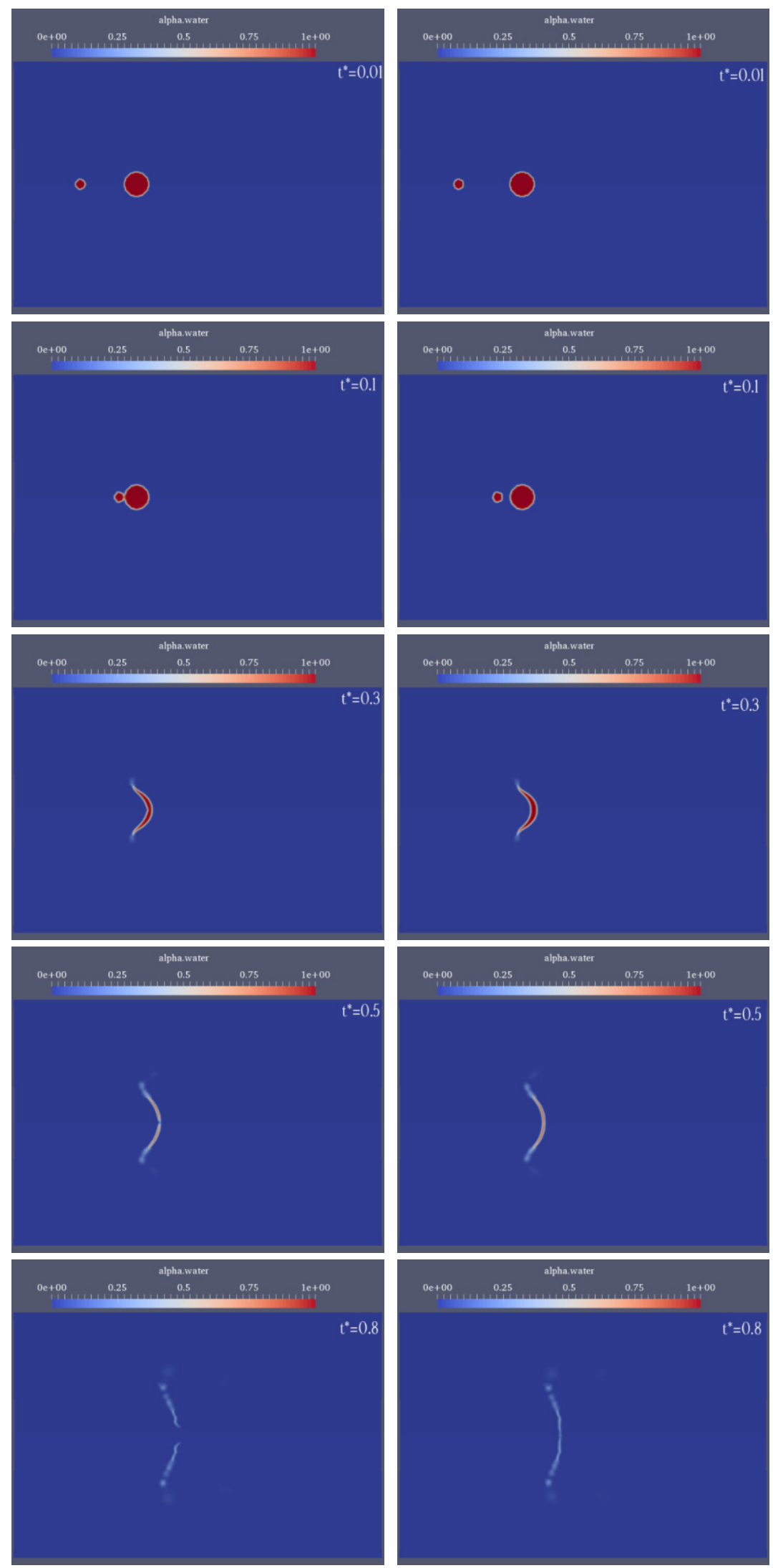

Fig. 9. Two-dimensional view of the two-respiratory droplet deformation at the penetrating and splashing stages. The dimensionless distance between the respiratory droplets is 5 (the first column) and 6 (the second column). The normalized diameter of the second respiratory droplet for the specified dimensionless distance is 0.4 . 
ORIGINAL RESEARCH
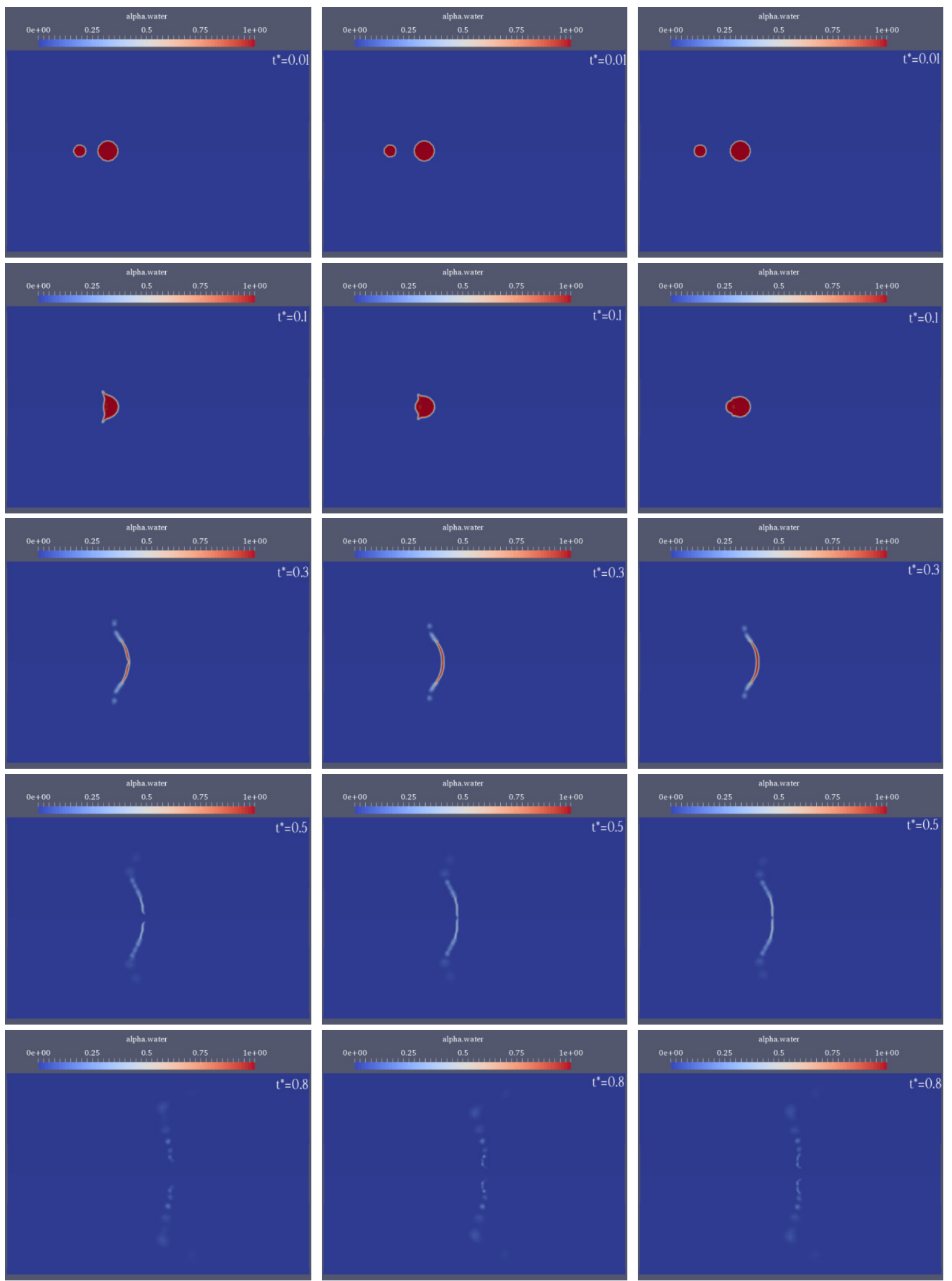

Fig. 10. Two-dimensional view of the two-respiratory droplet deformation at the penetrating and splashing stages. The dimensionless distance between the respiratory droplets is 2 (the first column), 3 (the second column), and 4 (the third column). The normalized diameter of the second respiratory droplet for the specified dimensionless distance is 0.6. 

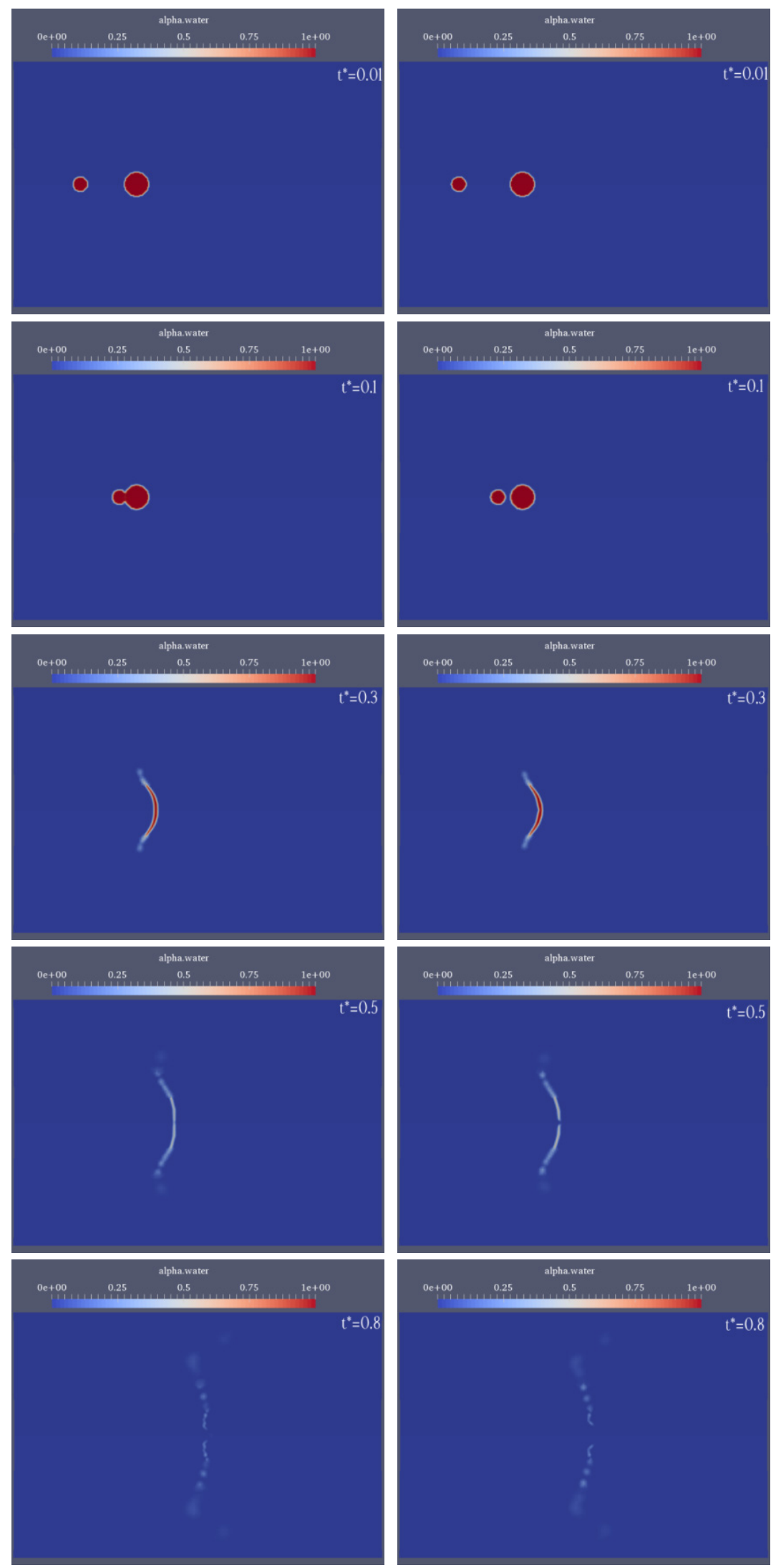

Fig. 11. Two-dimensional view of the two-respiratory droplet deformation at the penetrating and splashing stages. The dimensionless distance between the respiratory droplets is 5 (the first column) and 6 (the second column). The normalized diameter of the second respiratory droplet for the specified dimensionless distance is 0.6 . 
to that of the smaller respiratory droplet collision (Figs. 8 and 9 versus Figs. 10 and 11). Similar to the previous case, the pressure increases in the area of contact of two respiratory droplets. Due to the higher inertia of the larger secondary respiratory droplet, the high-pressure zone is more penetrated inside the first respiratory droplet after the collision takes place. One of the important results obtained from Figs. 8 and 9 (or Figs. 10 and 11) is that by decreasing the distance between two respiratory droplets, the time of penetration decreases, and the main respiratory droplet rapidly splashes.

The time needed for the secondary respiratory droplet to catch up with the main respiratory droplet is called the catch-up time shown by $t_{c}$. Based on the work of Zhao et al. (2019a), if the location of the two respiratory droplets is close to each other, the catch-up time and the breakup time would become shorter. The catch-up time is defined as:

$$
\begin{aligned}
& t_{c}=0.51 \sqrt{\frac{8}{3 C_{D_{0}}} I^{*}}\left(1-\frac{1-e^{-z}}{1+e^{-z}}\right)^{-1 / 2} \\
& z=4.7 \times 10^{-3} I^{*}+1.3 \frac{Y}{D_{0}}
\end{aligned}
$$

This correlation was obtained by performing the curve-fitting process on the data presented by Zhao et al. (2019a). $Y / D_{O}$ and $C_{D o}$ are the normalized distance of the secondary respiratory droplet in the $y$-direction and the drag coefficient of the main respiratory droplet, respectively. The catch-up times calculated for our case-using Eq. (7)-are listed in Table 2.

\subsubsection{Stage 2- Splashing}

Fig. $8\left(t^{*}=0.5\right.$ and 0.8$)$ demonstrates some snapshots of the two-dimensional two- respiratorydroplet breakup process at the second step called splashing. The main respiratory droplet transfigures to unequal and parallel coronas, which stretch in a radial direction. The origin of the birth of these coronas is the high momentum of the incoming secondary respiratory droplet, which is transferred to the main respiratory droplet without kinking at the boundary of the contact zone. The penetration of respiratory droplets into each other during an equal binary respiratory droplet collision has also been investigated by Passandideh-Fard and Roohi (2006), Acevedo-Malavé and García-Sucre (2011), Acevedo-Malavé (2012), and Zhao et al. (2019b). One of the main features of splashing is the disintegration of the thin liquid into smaller respiratory droplets at the early stages due to the manifestation of a thin liquid layer.

Figs. 12 and $13\left(\mathrm{t}^{*}=0.5\right.$ and 0.8$)$ show the details of the splashing of the main respiratory

Table 2. The catch-up time and breakup time calculated for the case of interaction of two respiratory droplets.

\begin{tabular}{lll}
\hline & Catch-up time $\left(t_{c}\right)$ & Breakup time $\left(t_{b}\right)$ \\
\hline$d^{*}=0.4, l^{*}=2$ & 0.250 & 0.730 \\
$d^{*}=0.4, l^{*}=3$ & 0.271 & 0.748 \\
$d^{*}=0.4, I^{*}=4$ & 0.293 & 0.769 \\
$d^{*}=0.4, l^{*}=5$ & 0.305 & 0.795 \\
$d^{*}=0.4, l^{*}=6$ & 0.381 & 0.861 \\
$d^{*}=0.6, l^{*}=2$ & 0.169 & 0.510 \\
$d^{*}=0.6, l^{*}=3$ & 0.174 & 0.531 \\
$d^{*}=0.6, l^{*}=4$ & 0.181 & 0.552 \\
$d^{*}=0.6, l^{*}=5$ & 0.188 & 0.574 \\
$d^{*}=0.6, l^{*}=6$ & 0.193 & 0.591 \\
$d^{*}=0.8, l^{*}=2$ & 0.128 & 0.400 \\
$d^{*}=0.8, l^{*}=3$ & 0.131 & 0.430 \\
$d^{*}=0.8, l^{*}=4$ & 0.136 & 0.451 \\
$d^{*}=0.8, l^{*}=5$ & 0.142 & 0.473 \\
$d^{*}=0.8, l^{*}=6$ & 0.148 & 0.485 \\
\hline
\end{tabular}



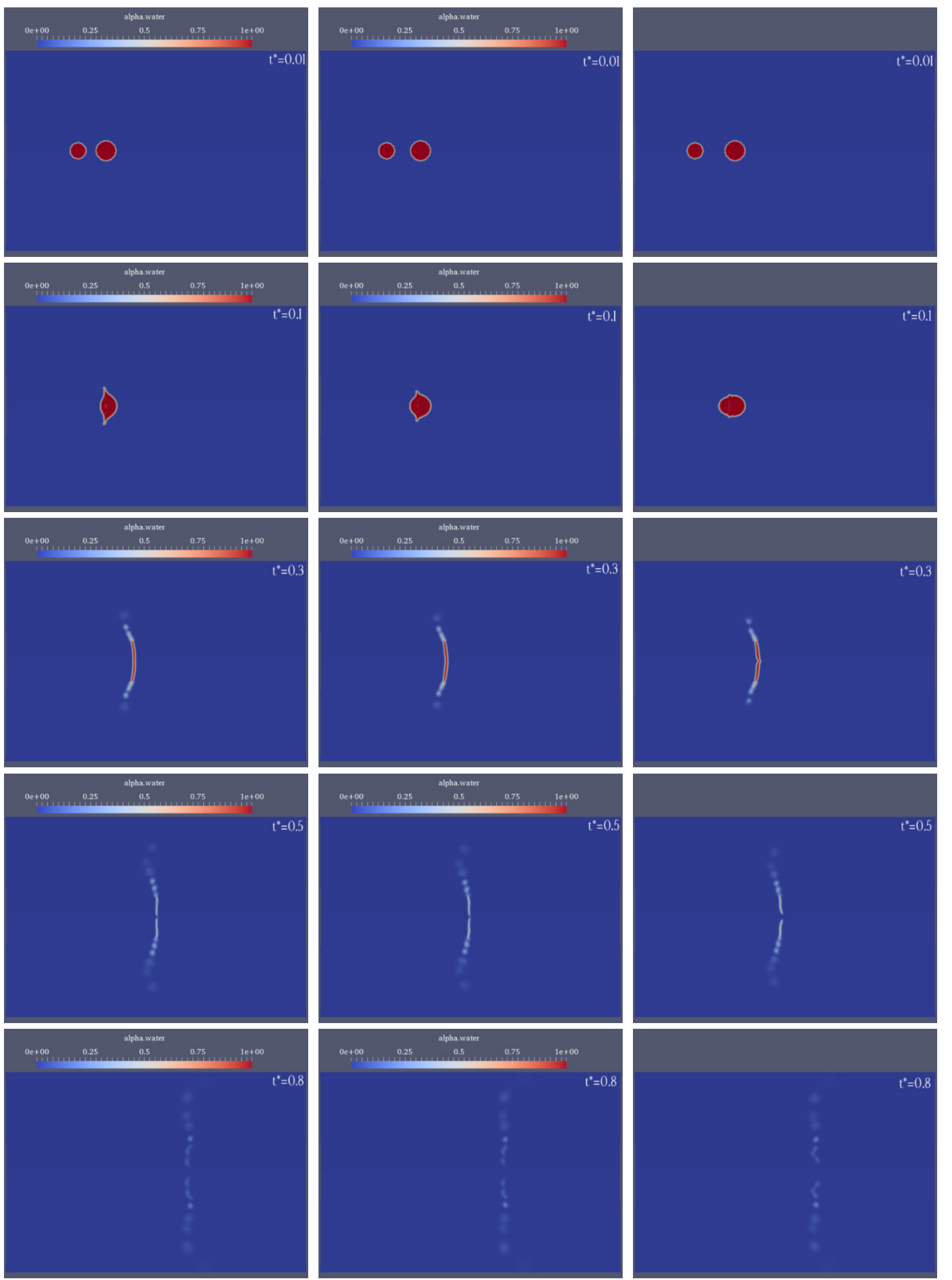

Fig. 12. Two-dimensional view of the two-respiratory droplet deformation at the penetrating and splashing stages. The dimensionless distance between the respiratory droplets is 2 (the first column), 3 (the second column), and 4 (the third column). The normalized diameter of the second respiratory droplet for the specified dimensionless distance is 0.8 . 

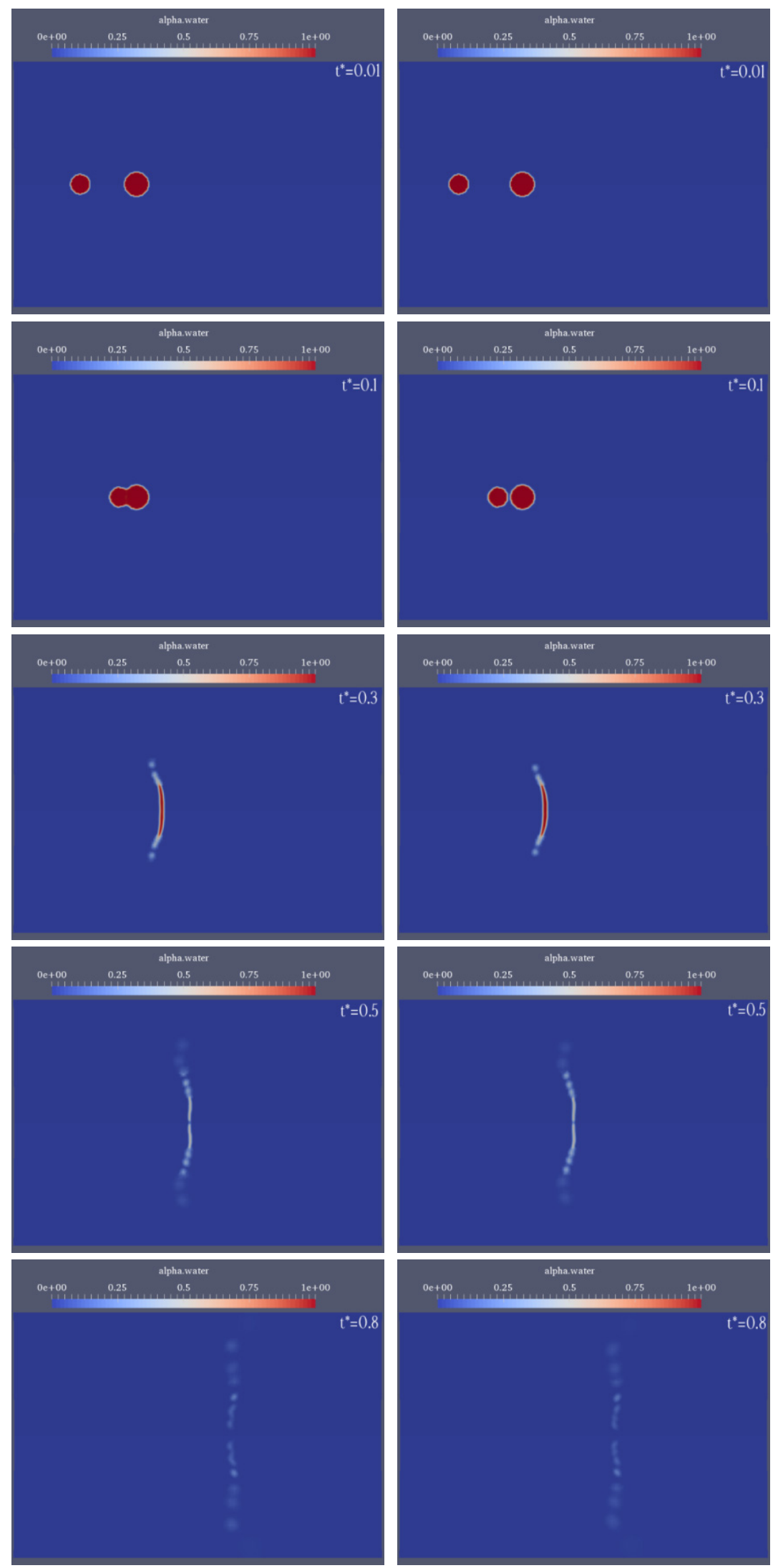

Fig. 13. Two-dimensional view of the two-respiratory droplet deformation at the penetrating and splashing stages. The dimensionless distance between the respiratory droplets is 5 (the first column) and 6 (the second column). The normalized diameter of the second respiratory droplet for the specified dimensionless distance is 0.8 . 
droplet after the collision of a larger secondary respiratory droplet, which leads to the creation of greater coronas with respect to the first case during a shorter catch-up time. Additionally, it is concluded that the initial distance between the respiratory droplets at this stage has a smaller influence on the flow pattern. The dimensionless breakup times of the main respiratory droplet with a normalized distance of 2 correspondings to the non-dimensional secondary respiratory droplet diameters of 0.6 and 0.4 are 0.64 and 0.71 , respectively. The breakup times increase to 0.712 and 0.731 for the case with a normalized distance of 3 .

Fig. 14 illustrates the variation of the aerosol width with respect to dimensionless distance. The results indicate that the aerosol width increases with decreases in the dimensionless distance. The secondary respiratory droplet suddenly stops and transfers its momentum to the stationary main respiratory droplet, and then the airborne cloud becomes visible during the mixing process. A low concentration of airborne is observed in the higher dimensionless distance between the respiratory droplets. In other words, the value of the transferred momentum to the main respiratory droplets decreases with an increase in the distance. Although the intermolecular forces greatly affect the surface tension, the variation of the aerosol width curves is related to the magnitude of the morphological forces acting on each respiratory droplet in the contact zone (Guan et al., 2018; Carrolo et al., 2019). Followings are three principal forces acting on the main respiratory droplet:

1- The drag force flattens the respiratory droplet.

2- The surface tension force exerts a resistive force against the flattening and restores the respiratory droplet back to its initial shape.

3- Inertia of the secondary respiratory droplet accelerates the splashing process.

The interplay between the influences of the surface tension force and the external aerodynamic forces (the drag force and the inertia) initiates the creation of an airborne cloud. It should be noted that the variation of the surface tension coefficient with temperature is considered in our study. However, the magnitude of the surface tension force varies with the curvature of the interface. Clearly, the value of the curvature is highly dependent on the morphological forces in the contact zone, the relative humidity, and the ambient temperature. By decreasing the radius of curvature, the surface tension force increases in accordance with Tolman's hypothesis (Bartell, 2001; Shneider and Pekker, 2019).

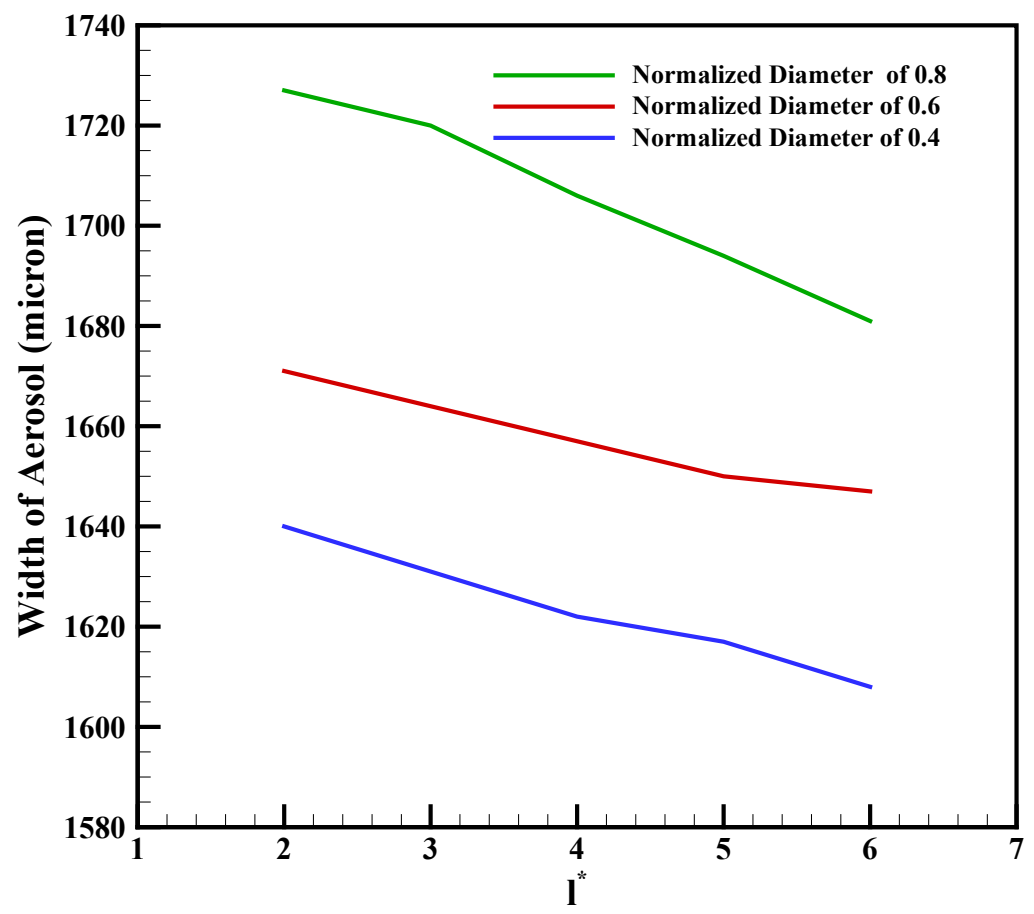

Fig. 14. The width of aerosol variation versus dimensionless distance at high-speed wind of $50 \mathrm{~km} \mathrm{~h}^{-1}$ for the case of two-respiratory droplet interaction. 


\subsection{CASE B: THE THREE-RESPIRATORY- DROPLET COLLISION}

Considering the collision of two secondary respiratory droplets with the main respiratory droplet, the breakup characteristics and the morphology of the main respiratory droplet in connection with other smaller respiratory droplets will be analyzed in this section. To generate the numerical results, four different positions for the smaller respiratory droplets were selected along with the following geometrical parameters:

- $\quad$ Case a: The dimensionless diameter of both small respiratory droplets is 0.4 .

- $\quad$ Case b: The dimensionless diameter of both small respiratory droplets is 0.6.

- $\quad$ Case c: The diameter of the closest respiratory droplet to the main respiratory droplet is 0.6, and the diameter of the other respiratory droplet is 0.4 .

- $\quad$ Case $\mathrm{d}$ : The diameter of the closest respiratory droplet to the main respiratory droplet is 0.4 , and the diameter of the other respiratory droplet is 0.6 .

All three respiratory droplets are in tandem arrangement along with the direction of the incoming high-speed wind flow. Similar to the previous case, the breakup and deformation of the main respiratory droplet are governed by two steps.

\subsubsection{Stage 1- Penetration}

Figs. 15 and $16\left(\mathrm{t}^{*}=0.01-0.3\right)$ present snapshots of the two-dimensional three-respiratory droplet collision at the penetrating stage. The major difference between the two respiratory droplets and the three respiratory droplet deformations is that in the three respiratory droplet case, the last respiratory droplet hits the bowl-like shape of the liquid, which is formed after penetration of the first respiratory droplet. Therefore, the next collision takes place between the third respiratory droplet and the bowl-like structure with extremely thin walls, which leads to the verge of the splash. It is seen that due to the larger diameter of the second respiratory droplet, the thickness of the bowl-like shape and its diameter is greater in "Case b", and the pressure reduces at the tip of the bowl. Additionally, a high-pressure zone is seen near the lee side of the first respiratory droplet due to the effect of the collision of the two other respiratory droplets.

The three respiratory-drop collision catch-up times for "Cases a to d" are calculated based on the final collision using Eq. (7), and the results are presented in Table 3. The largest and shortest collision times correspond to "Case a" containing small respiratory droplets and "Case $d$ " with large respiratory droplets, respectively. This trend stems from the higher inertia of the incoming respiratory droplets in "Case a", which speeds up the collision process.

\subsubsection{Stage 2- Splashing}

As shown in Figs. 16 and $17\left(t^{*}=0.5\right.$ and 0.8$)$, following the previous stage, the main respiratory droplet converts the bowl-shaped part of the liquid into unequal and parallel coronas. This stage of the three-respiratory-droplet breakup is similar to the final stage of the two-respiratory-droplet deformation process. As the material strips off the bowl liquid edge by the surrounding highspeed wind flow, the thickness of the coronas rapidly decreases. The smaller rate of feeding the film with transferred momentum causes the thin bowl-like liquid to splash at a shorter time. The dimensionless mixing times of the main respiratory droplet for Cases $a, b, c$, and $d$ are 0.387, $0.301,0.357$, and 0.376 , respectively. The presence of small neighboring respiratory droplets reduces the breakup time by approximately $5 \%$. However, the presence of two small neighboring respiratory droplets results in a change of approximately $7 \%$ in the catch-up time.

\section{THE EFFECTS OF WIND VELOCITY ON AEROSOL CLOUD}

Our numerical study revealed how micro-respiratory droplets-during coughs and sneezescan be dispersed in aerosol clouds, precisely the same as the real condition. Figs. 17 and 18 show the width of aerosol-cloud variation with the various wind velocities for two- and three-respiratory droplet splashing, respectively. First, the respiratory droplets expelled from the mouth are mostly affected by the inertia force and are moving along the direction of the high-speed wind velocity. These respiratory droplets interact with each other and convert to aerosol clouds under highspeed wind conditions. The results show that the aerosol width increases with an increase in the high-speed wind velocity. During the high-speed wind flow, some of the larger respiratory droplets 

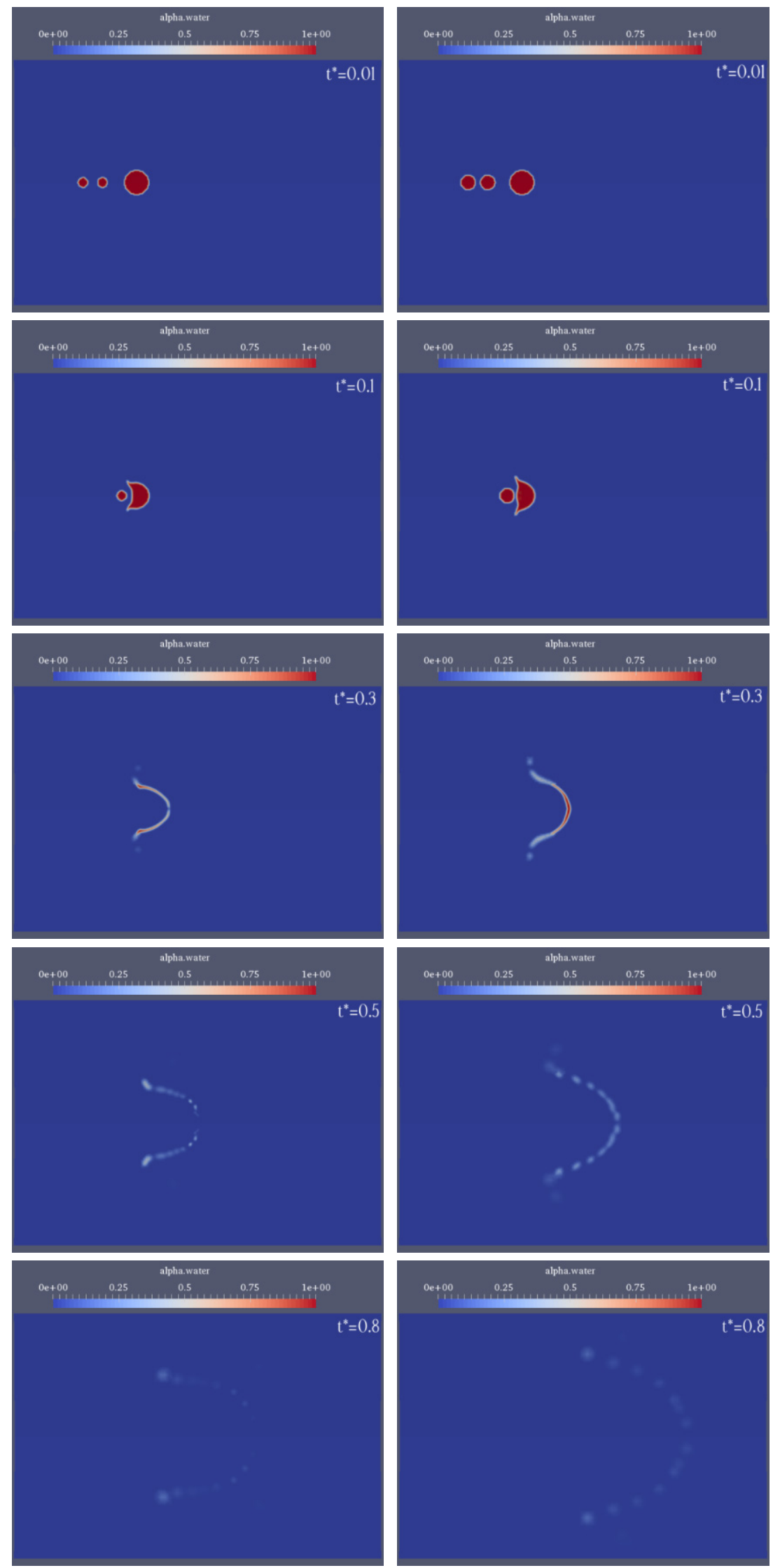

Fig. 15. Two-dimensional view of the three-respiratory-droplet breakup in high-speed wind flow at the penetrating and splashing stages. The first and second columns of each time correspond to "Cases a and b", respectively. 

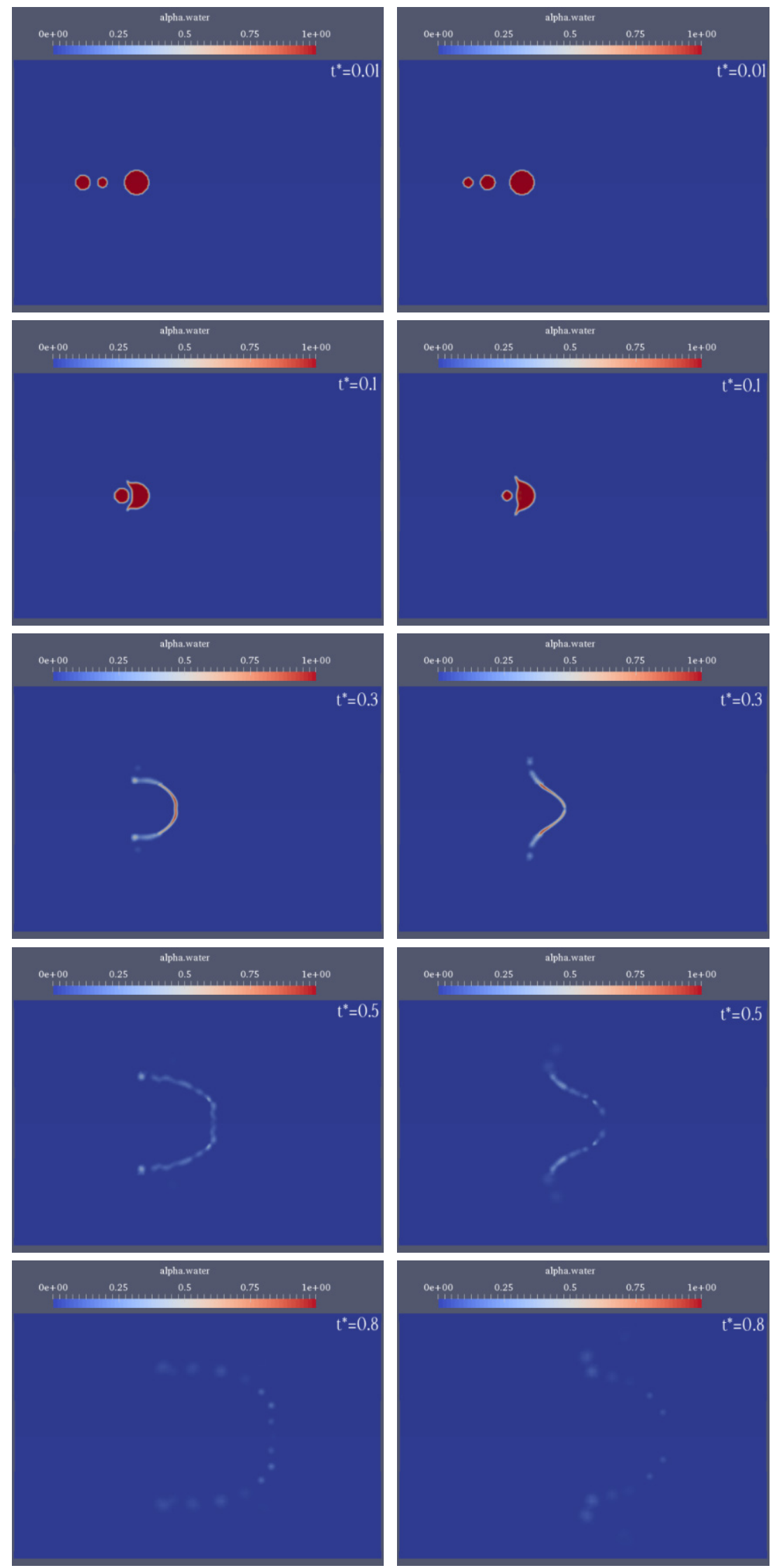

Fig. 16. Two-dimensional view of the three-respiratory-droplet breakup in high-speed wind flow at the penetrating and splashing stages. The first and second columns of each time correspond to "Cases c and d", respectively. 
Table 3. The catch-up time calculated for the case of interaction of two respiratory droplets.

\begin{tabular}{lll}
\hline & Catch up time $\left(t_{c}\right)$ & Breakup time $\left(t_{b}\right)$ \\
\hline Case a & 0.123 & 0.387 \\
Case b & 0.107 & 0.301 \\
Case c & 0.114 & 0.357 \\
Case d & 0.120 & 0.376 \\
\hline
\end{tabular}

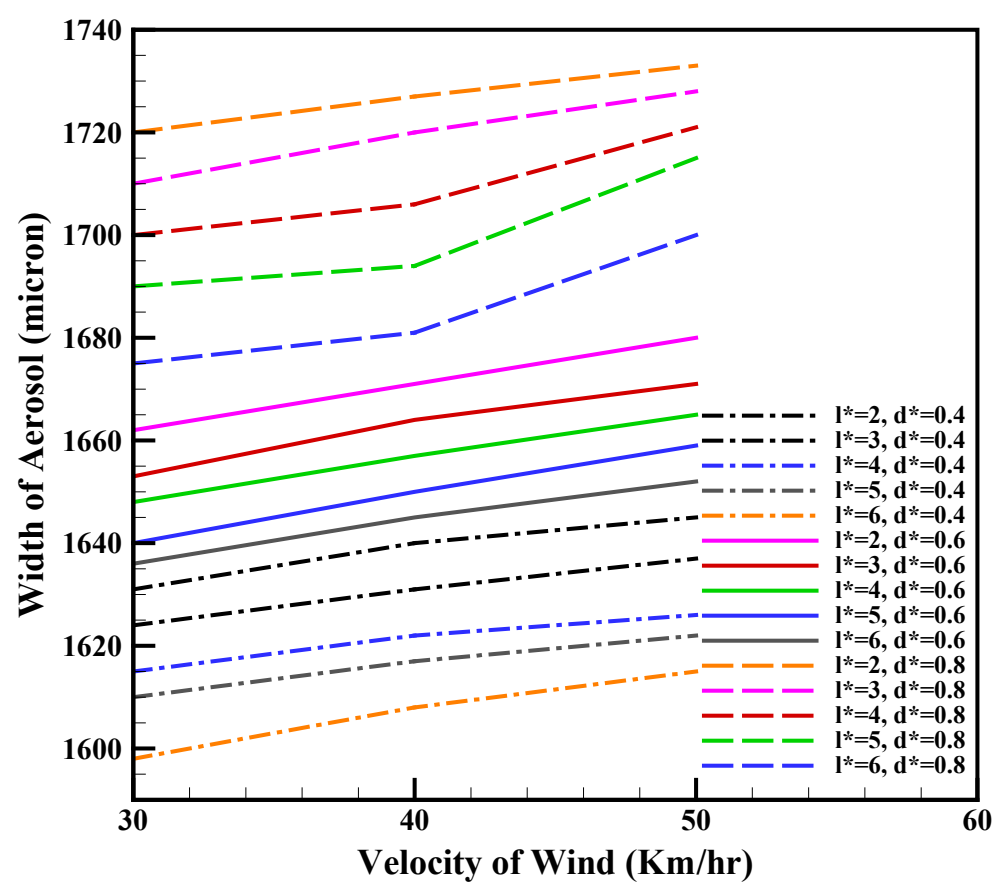

Fig. 17. Variation of the wind velocity on the width of the aerosol corresponding to the tworespiratory droplet case.

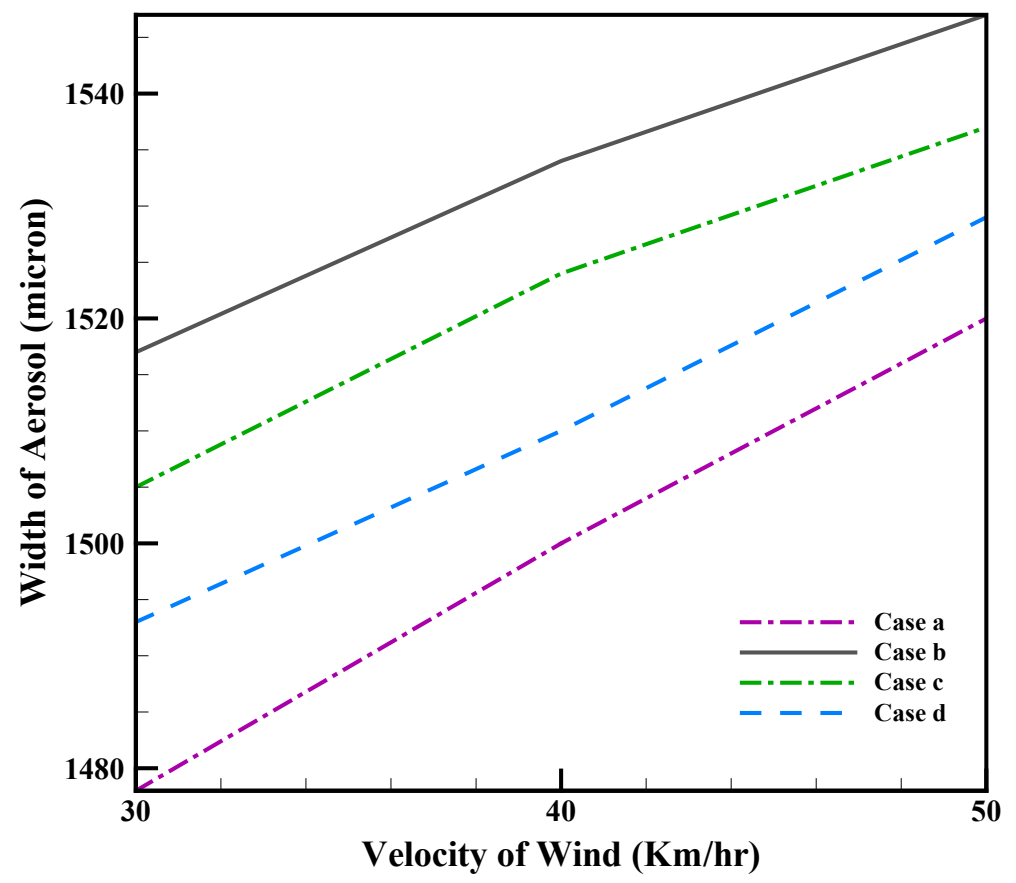

Fig. 18. Variation of the wind velocity on the width of the aerosol corresponding to the three respiratory droplet case. 
fell on the floor, and some of them converted to smaller droplets during violent interaction. The smaller particles of respiratory droplets under high-speed wind conditions continue to fluctuate and take considerable time to reach the ground. The high-speed wind velocity increases the concentration of the airborne and may influence the infection's intensity and progression.

\section{THE EFFECTS OF THE RELATIVE HUMIDITY AND AMBIENT TEMPERATURE ON AEROSOL CLOUD}

Respiratory droplets can convert to clouds of airborne, viruses, and very small respiratory droplets on the high-speed wind flow by collision and breakup of the larger respiratory droplets. The width and thickness of the created aerosol vary in different situations. To determine the effect of the climate on the exhaled respiratory droplets, we considered the ambient temperature and the ambient humidity. We have previously studied the effects of $\mathrm{RH}$ and temperature on the survival of SARS-CoV-2 (Rezaei et al., 2020). Rezaei et al. (2020) demonstrated that SARS-CoV-2 rapidly disappears in a condition with a temperature range of $50^{\circ} \mathrm{C}-80^{\circ} \mathrm{C}$ and a relative humidity range of $40 \%-50 \%$. However, the current study focused on the outdoor transmission of SARSCoV-2 at a windy condition, which differs from the indoor situation with zero-wind velocity. The mechanism of the effect of relative humidity on the virus survival is important and the climate situation should be considered to achieve precise results. Based on Moriyama et al. (2020) and Ahlawat et al. (2020) studies, relative humidity of 40-60\% was found to be appropriate for limiting respiratory virus transmission in indoor places. The present study showed that a relative humidity level of $40-70 \%$ at high-speed wind velocity is not appropriate for the inactivation of viruses inside micro-droplets. This contradiction originated from parameters such as high-speed wind velocity, Water activity, surface inactivation, and salt toxicity in the virus's persistence in experimental studies. (Yang et al., 2012). Various researches such as Dbouk and Drikakis (2020c), Vinoj et al. (2020) affirm our results. In this study, by adding of conservation of species for the specific humidity to the VOF solver, the effect of RH on the width of the aerosol. Figs. 19-22 show the variation of the aerosol width with the $\mathrm{RH}$ and temperature for all simulation cases during the collision. Our results indicate that exposing exhaled respiratory droplets to a higher ambient temperature and lower $\mathrm{RH}$ reduces the aerosol size. At higher ambient temperature and lower humidity (Dbouk and

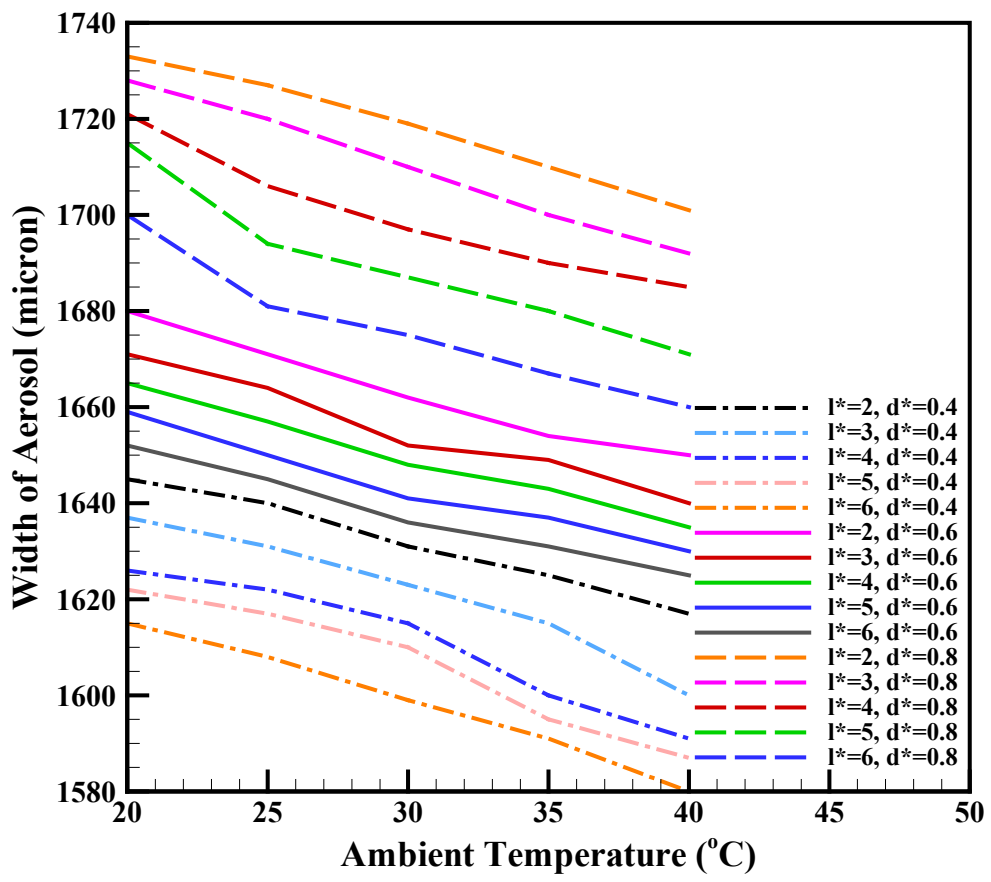

Fig. 19. Variation of the ambient temperature on the width of the aerosol corresponding to the two-respiratory droplet case. 


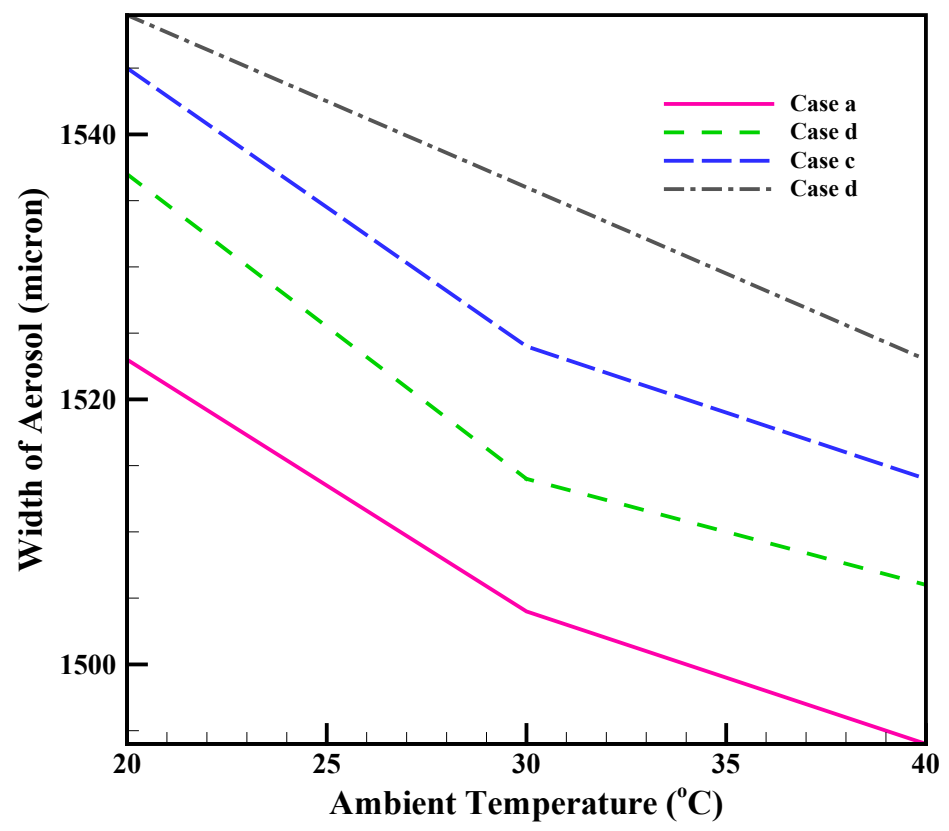

Fig. 20. Variation of the ambient temperature on the width of the aerosol corresponding to the three-respiratory droplet case.

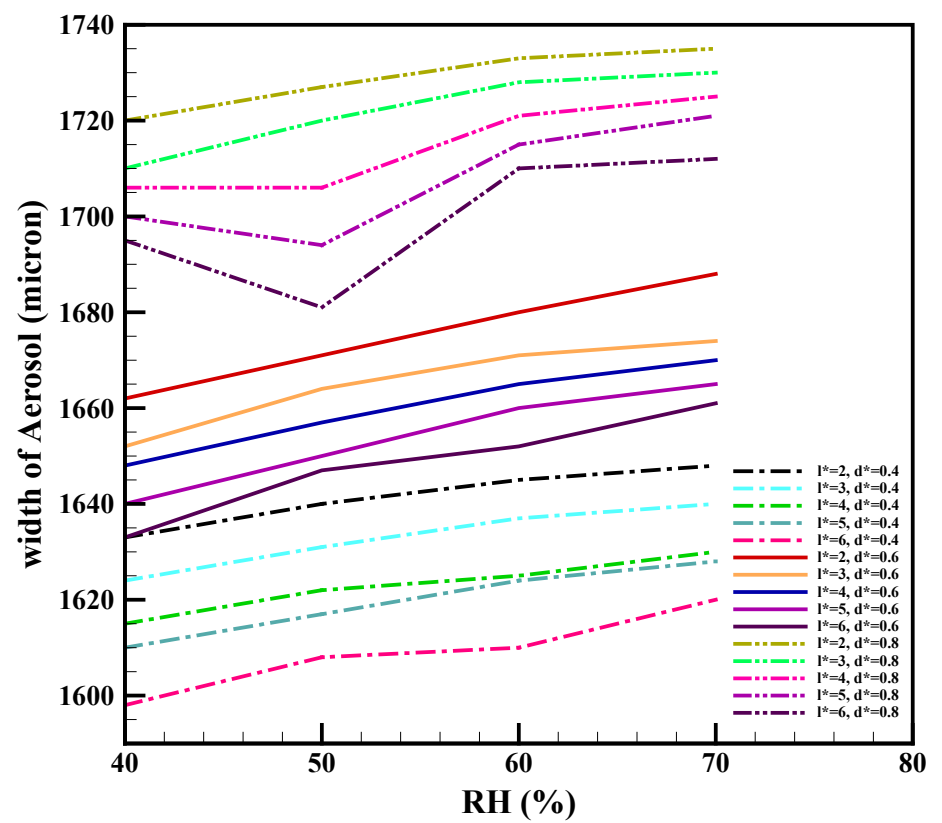

Fig. 21. Variation of the ambient relative humidity $(\mathrm{RH})$ on the width of the aerosol corresponding to the two-respiratory droplet case.

Drikakis, 2020c), the study concluded that protein is a crucial component of the virus structure. This protein is very unstable in the mentioned ambient situation, similar to the higher wind velocity.

\section{THE ROLE OF FACE MASK WEARING IN THE CONTROL OF COVID-19}

Nowadays, the crucial protective effect of face mask against SARS-CoV-2 has been highlighted by all scientific authorities (Feng et al., 2020; Pei et al., 2020). The spreading of viruses such as a 


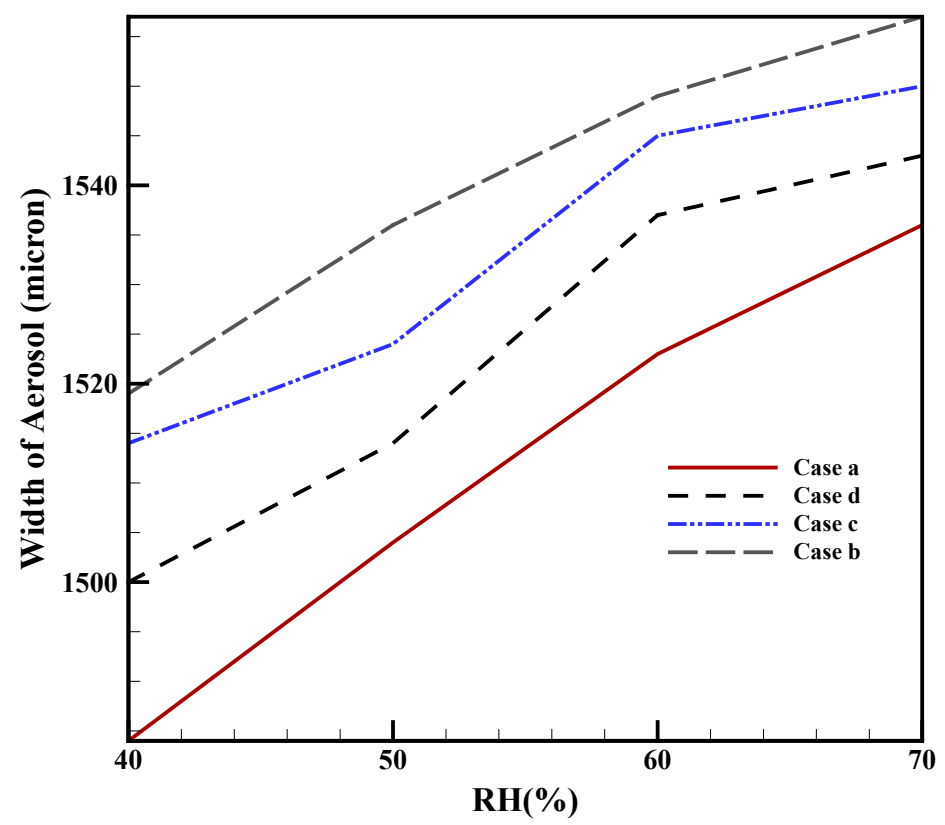

Fig. 22. Variation of the ambient relative humidity $(\mathrm{RH})$ on the width of the aerosol corresponding to the three respiratory droplet case.

SARS-CoV-2 increase when the breakup time of the respiratory droplets decreases to 0.301 . The number of respiratory droplet concentrations in the ambient air depends on the usage of the face mask during sneezing and coughing. It is important to note that these respiratory droplets collide with neighboring respiratory droplets under high-speed wind conditions and convert to very small airborne respiratory particles (approximately 500 nanometers). These particles (airborne SARS-CoV-2) significantly increase the risk of transmission of COVID 19. By wearing a face mask, the number of respiratory droplets-upon sneezing or coughing-decrease, that in turn, decreases the formation of very small airborne particles and infectivity of COVID-19. In the following, we have provided learning tips for public health impact and SARS-CoV-2 control in outdoor transmission:

- Wearing a face mask and keeping the social distance in outdoor environments are suggested.

- If a face mask is not used (for any reason), try to cover the two openings of the airway (i.e., the nose and mouth) in a windy condition.

- Apply a dehumidifier in air supply ducts in heating and ventilating systems.

\section{CONCLUSIONS}

Respiratory flow-expelled by a human during sneezing, coughing, or loudly talking-consists of a poly-dimension/distance collection of respiratory droplets/particles. In this article, we have presented a novel technique for the collision, deformation, and breakup of respiratory droplets under high-speed wind conditions. We considered the two- and three-respiratory droplets in these situations to calculate the breakup and catch-up time and study the morphology of the respiratory droplet in connection with other smaller respiratory droplets. We demonstrated the importance of face mask usage from a novel point of view. The mechanisms of the breakup, deformation, and collision of respiratory droplets in a high-speed wind stream of air were investigated. Three different positions of the respiratory droplets were selected, and the simulation of highly compressible two-phase flow was performed using the OpenFOAM C++ libraries. After validating the numerical results using the experimental data, several conclusions regarding the different features and regimes of respiratory droplet breakup and COVID-19 spreading were obtained as follows:

1) The use of the face mask will provide good prevention of respiratory droplet transmission during sneezing, coughing, or loudly talking in high-speed wind conditions.

2) The diameters and the distances between the expelled respiratory droplets (concentration of SARS-CoV-2) during sneezing and coughing depend on the usage of the face mask. 
3) The spreading of the SARS-CoV-2 aerosol depends on the respiratory droplet breakup time. The spreading of SARS-CoV-2 increases with a decrease in respiratory droplet breakup time.

4) The deformation process for the one-respiratory droplet case is classified into three stages.

- The appearance of the surface instability,

- $\quad$ Flattening the respiratory droplet,

- Entrainment from the liquid sheet.

5) The deformation process for two- and three-respiratory-droplet collisions is categorized into two steps:

- $\quad$ penetration of the smaller respiratory droplets into the main respiratory droplet,

- $\quad$ splashing of the main respiratory droplet.

6) The thickness of the deformed main respiratory droplet and the volume fraction of the liquid have been taken as two criteria to define the dimensionless breakup time. As a result, the normalized breakup time of the respiratory droplet at the high-speed wind has been computed to be approximately 0.301 and 0.4 for three- and two-respiratory droplet interactions, respectively.

7) The temporal variation of the center of mass velocity of the main respiratory droplet has been studied for two- and three-respiratory droplet cases. The velocity increases with time until a minimum point appears in the curve for each collision. The analysis of the results showed that this pattern of change depends on the morphological force acting on the respiratory droplets during the mixing process.

8) The advanced stages of the breakup of the resulting respiratory particle clouds have been observed, and a clear definition and quantification of the respiratory droplet breakup times have been offered.

9) As a result, with an increase in the wind velocity and the ambient relative humidity, the spreading of viruses increases. Also, with a decrease in the ambient temperature, mentioned spreading increases.

\section{NOMENCLATURE}

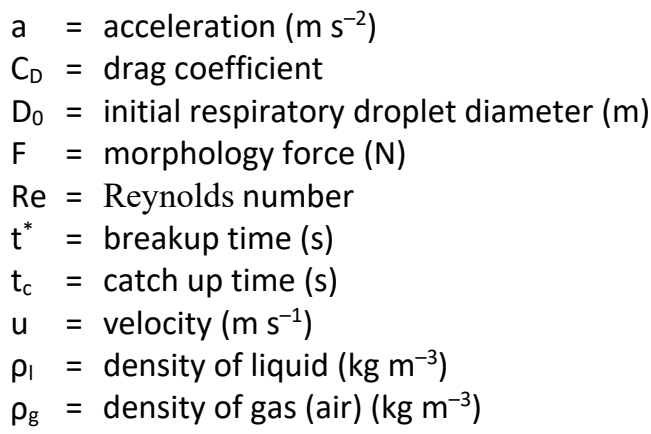

\section{Subscripts \\ $g$ = gas \\ $l=$ liquid \\ $c=$ catch up}

\section{DATA AVAILABILITY}

The data that support the findings of this study are available from the corresponding author upon reasonable request.

\section{ACKNOWLEDGEMENT}

The publishing fee is supported by the Grants-in-Aid for the Excellent Young Researcher of the Ministry of Education of Japan (MEXT). 


\section{REFERENCES}

Acevedo-Malavé, A. (2012). Hydrodynamics coalescence collision of three liquid drops in 3D with smoothed particle hydrodynamics. AIP Adv. 2, 042106. https://doi.org/10.1063/1.4757966

Acevedo-Malavé, A., García-Sucre, M. (2011). Coalescence collision of liquid drops I: Off-center collisions of equal-size drops. AIP Adv. 1, 032117. https://doi.org/10.1063/1.3624553

Ahlawat, A., Wiedensohler, A., Mishra, S.K. (2020). An overview on the role of relative humidity in airborne transmission of SARS-CoV-2 in indoor environments. Aerosol Air Qual. Res. 20(9), 1856-1861. https://doi.org/10.4209/aaqr.2020.06.0302

Ameri, A., Rahnama, N., Bozorgmehr, R., Mokhtari, M., Farahbakhsh, M., Nabavi, M., Shoaei, S.D., Izadi, H., Yousefi Kashi, A.S., Dehbaneh, H.S., Taghizadeh-Hesary, F. (2020). Low-dose wholelung irradiation for COVID-19 pneumonia: Short course results. Int. J. Radiat. Oncol. Biol. Phys. 108, 1134-1139. https://doi.org/10.1016/j.ijrobp.2020.07.026

Ameri, A., Ameri, P., Rahnama, N., Mokhtari, M., Sedaghat, M., Hadavand, F., Bozorgmehr, R., Haghighi, M., Taghizadeh-Hesary, F. (2021). Low-dose whole-lung irradiation for COVID-19 pneumonia: Final results of a pilot study. Int. J. Radiat. Oncol. Biol. Phys. 109, 859-866. https://doi.org/10.1016/j.ijrobp.2020.11.065

Asadi, S., Wexler, A.S., Cappa, C.D., Barreda, S., Bouvier, N.M., Ristenpart, W.D. (2019). Aerosol emission and superemission during human speech increase with voice loudness. Sci. Rep. 9, 2348. https://doi.org/10.1038/s41598-019-38808-z

Bartell, L.S. (2001). Tolman's $\delta$, surface curvature, compressibility effects, and the free energy of drops. J. Phys. Chem. B 105, 11615-11618. https://doi.org/10.1021/jp011028f

Berberović, E., van Hinsberg, N.P., Jakirlić, S., Roisman, I.V., Tropea, C. (2009). Drop impact onto a liquid layer of finite thickness: Dynamics of the cavity evolution. Phys. Rev. E 79, 036306. https://doi.org/10.1103/PhysRevE.79.036306

Bhardwaj, R., Agrawal, A. (2020). Tailoring surface wettability to reduce chances of infection of COVID-19 by a respiratory droplet and to improve the effectiveness of personal protection equipment. Phys. Fluids 32, 081702. https://doi.org/10.1063/5.0020249

Bourouiba, L. (2020). Turbulent gas clouds and respiratory pathogen emissions: Potential implications for reducing transmission of COVID-19. JAMA 323, 1837-1838. https://doi.org/10. 1001/jama.2020.4756

Carelli, P. (2020). A physicist's approach to COVID-19 transmission via expiratory droplets. Med. Hypotheses 144, 109997. https://doi.org/10.1016/j.mehy.2020.109997

Carrolo, G., Ribeiro, D., Barata, J.M., Silva, A.R. (2019). Aerodynamic Breakup of a Single Droplet due to a Crossflowed Airstream, in: AIAA Scitech 2019 Forum, American Institute of Aeronautics and Astronautics. https://doi.org/10.2514/6.2019-0628

Chaudhuri, S., Basu, S., Kabi, P., Unni, V. R., Saha, A. (2020). Modeling the role of respiratory droplets in Covid-19 type pandemics. Phys. Fluids 32, 063309. https://doi.org/10.1063/5.0015 984

Chen, L.D. (2020). Effects of ambient temperature and humidity on droplet lifetime - A perspective of exhalation sneeze droplets with COVID-19 virus transmission. Int. J. Hyg. Environ. Health 229, 113568. https://doi.org/10.1016/j.ijheh.2020.113568

Chen, X., Zhou, X., Xia, X., Xie, X., Lu, P., Feng, Y. (2020). Modeling of the transport, hygroscopic growth, and deposition of multi-component droplets in a simplified airway with realistic thermal boundary conditions. J. Aerosol Sci. 151, 105626. https://doi.org/10.1016/j.jaerosci.2 020.105626

Dbouk, T., Drikakis, D. (2020a). On coughing and airborne droplet transmission to humans. Phys. Fluids 32, 053310. https://doi.org/10.1063/5.0011960

Dbouk, T., Drikakis, D. (2020b). On respiratory droplets and face masks. Phys. Fluids 32, 063303. https://doi.org/10.1063/5.0015044

Dbouk, T., Drikakis, D. (2020c). Weather impact on airborne coronavirus survival. Phys. Fluids 32, 093312. https://doi.org/10.1063/5.0024272

Deshpande, S.S., Anumolu, L., Trujillo, M.F. (2012). Evaluating the performance of the two-phase flow solver interFoam. Comput. Sci. Discovery 5, 014016. https://doi.org/10.1088/17494699/5/1/014016 
Drossinos, Y., Stilianakis, N.I. (2020). What aerosol physics tells us about airborne pathogen transmission. Aerosol Sci. Technol. 54, 639-643. https://doi.org/10.1080/02786826.2020.175 1055

Feng, S., Shen, C., Xia, N., Song, W., Fan, M., Cowling, B.J. (2020). Rational use of face masks in the COVID-19 pandemic. Lancet Respir. Med. 8, 434-436. https://doi.org/10.1016/S22132600(20)30134-X

Guan, B., Liu, Y., Wen, C.Y., Shen, H. (2018). Numerical study on liquid droplet internal flow under shock impact. AIAA J. 56, 3382-3387. https://doi.org/10.2514/1.J057134

Guildenbecher, D.R., López-Rivera, C., Sojka, P.E. (2009). Secondary atomization. Exp. Fluids 46, 371. https://doi.org/10.1007/s00348-008-0593-2

Hsiao, T.C., Chuang, H.C., Griffith, S.M., Chen, S.J., Young, L.H. (2020). COVID-19: An aerosol's point of view from expiration to transmission to viral-mechanism. Aerosol Air Qual. Res. 20, 905-910. https://doi.org/10.4209/aaqr.2020.04.0154

Johns Hopkins University and Medicine (2020). COVID-19 mortality rate. https://coronavir us.jhu.edu/map.html (accessed 12 September 2020).

Li, Y.Y., Wang, J.X., Chen, X. (2020). Can a toilet promote virus transmission? From a fluid dynamics perspective. Phys. Fluids 32, 065107. https://doi.org/10.1063/5.0013318

Moriyama, M., Hugentobler, W.J., Iwasaki, A. (2020). Seasonality of respiratory viral infections. Annu. Rev. Virol. 7, 83-101. https://doi.org/10.1146/annurev-virology-012420-022445

National Academies of Sciences, Engineering, and Medicine (2020). Rapid expert consultation on SARS-CoV-2 viral shedding and antibody response for the COVID-19 pandemic, April 8, 2020, pp. 1-7. https://doi.org/10.17226/25774

Nazari, A., Jafari, M., Rezaei, N., Taghizadeh-Hesary, F., Taghizadeh-Hesary, F. (2021). Jet fans in the underground car parking areas and virus transmission. Phys. Fluids 33, 013603. https://doi.org/10.1063/5.0033557

OpenFOAM Foundation Ltd. (2016a). OpenFOAM - the open source CFD toolbox - Programmers' guide. https://www.openfoam.com/

OpenFOAM Foundation Ltd. (2016b). OpenFOAM - the open source CFD toolbox - User's guide. https://www.openfoam.com/

Passandideh-Fard, M., Roohi, E. (2006). Coalescence collision of two droplets: Bubble entrapment and the effects of important parameters. 14th Annual (International) Mechanical Engineering Conference, Isfahan. https://civilica.com/doc/27993/

Pei, C., Ou, Q., Kim, S.C., Chen, S.C., Pui, D.Y.H. (2020). Alternative Face masks made of common materials for general public: Fractional filtration efficiency and breathability perspective. Aerosol Air Qual. Res. 20, 2581-2591. https://doi.org/10.4209/aaqr.2020.07.0423

Plog, J., Wu, J., Dias, Y.J., Mashayek, F., Cooper, L.F., Yarin, A.L. (2020). Reopening dentistry after COVID-19: Complete suppression of aerosolization in dental procedures by viscoelastic Medusa Gorgo. Phys. Fluids 32, 083111. https://doi.org/10.1063/5.0021476

Rakhsha, A., Azghandi, S., Taghizadeh-Hesary, F. (2020). COVID-19 pandemic and patients with cancer: The protocol of a Clinical Oncology center in Tehran, Iran. Rep. Pract. Oncol. Radiother. 25, 765-767. https://doi.org/10.1016/j.rpor.2020.07.001

Rezaei, N., Jafari, M., Nazari, A., Salehi, S., Talati, F., Torab, R., Nejad-Rahim, R. (2020). A novel methodology and new concept of SARS-CoV-2 elimination in heating and ventilating air conditioning systems using waste heat recovery. AIP Adv. 10, 085308. https://doi.org/10.1063/ 5.0021575

Riediker, M., Morawska, L. (2020). Low exhaled breath droplet formation may explain why children are poor SARS-CoV-2 transmitters. Aerosol Air Qual. Res. 20, 1513-1515. https://doi.org/10.4209/aaqr.2020.06.0304

Rodrigues, C., Barata, J., Silva, A. (2016). Modeling of droplet deformation and breakup. J. Propuls. Power 32, 698-706. https://doi.org/10.2514/1.B35819

Schroter, R.C. (2020). Social distancing for covid-19: Is 2 metres far enough? BMJ 369, m2010. https://doi.org/10.1136/bmj.m2010

Shneider, M.N., Pekker, M. (2019). Surface tension of small bubbles and droplets and the cavitation threshold. arXiv preprint arXiv:1901.04329. https://arxiv.org/abs/1901.04329

Siavashpour, Z., Taghizadeh-Hesary, F., Rakhsha, A. (2020). Recommendations on management of locally advanced rectal cancer during the COVID-19 pandemic: An Iranian consensus. J 
Gastrointest. Canc. 51, 800-804. https://doi.org/10.1007/s12029-020-00454-4

Simpkins, P.G., Bales, E.L. (1972). Water-drop response to sudden accelerations. J. Fluid Mech. 55, 629-639. https://doi.org/10.1017/S0022112072002058

Smits, A.J., Dussauge, J.P. (2006). Turbulent shear layers in supersonic flow. Springer Science \& Business Media. https://www.springer.com/gp/book/9780387261409

Sun, C., Zhai, Z. (2020). The efficacy of social distance and ventilation effectiveness in preventing COVID-19 transmission. Sustainable Cities Soc. 62, 102390. https://doi.org/10.1016/j.scs.2020. 102390

Taghizadeh-Hesary, F., Akbari, H. (2020). The powerful immune system against powerful COVID-19: A hypothesis. Med. Hypotheses 140, 109762. https://doi.org/10.1016/j.mehy.2020.109762

Tryggvason, G., Bunner, B., Esmaeeli, A., Juric, D., Al-Rawahi, N., Tauber, W., Han, J., Nas, S., Jan, Y.J. (2001). A front-tracking method for the computations of multiphase flow. J. Comput. Phys. 169, 708-759. https://doi.org/10.1006/jcph.2001.6726

Vinoj, V., Gopinath, N., Landu, K., Behera, B., Mishra, B. (2020). The COVID-19 spread in India and its dependence on temperature and relative humidity. Preprints 2020, 2020070082. https://www.preprints.org/manuscript/202007.0082/v1

Wang, D., Sun, B.C., Wang, J.X., Zhou, Y.Y., Chen, Z.W., Fang, Y., Yue, W.H., Liu, S.M., Liu, K.Y., Zeng, X.F., Chu, G.W., Chen, J.F. (2020). Can masks be reused after hot water decontamination during the COVID-19 pandemic? Engineering 6, 1115-1121. https://doi.org/10.1016/j.eng.202 0.05 .016

Wilson, N.M., Norton, A., Young, F.P., Collins, D.W. (2020). Airborne transmission of severe acute respiratory syndrome coronavirus- 2 to healthcare workers: A narrative review. Anaesthesia 75, 1086-1095. https://doi.org/10.1111/anae.15093

Xie, X., Li, Y., Chwang, A.T.Y., Ho, P.L., Seto, W.H. (2007). How far droplets can move in indoor environments-revisiting the Wells evaporation-falling curve. Indoor Air 17, 211-225. https://doi.org/10.1111/j.1600-0668.2007.00469.x

Xie, X., Li, Y., Sun, H., Liu, L. (2009). Exhaled droplets due to talking and coughing. J. R. Soc. Interface 6, S703-S714. https://doi.org/10.1098/rsif.2009.0388.focus

Yan, J., Grantham, M., Pantelic, J., de Mesquita, P.J.B., Albert, B., Liu, F., Ehrman, S., Milton, D.K., Consortium, E. (2018). Infectious virus in exhaled breath of symptomatic seasonal influenza cases from a college community. PNAS 115, 1081-1086. https://doi.org/10.1073/pnas.171656 1115

Yang, W., Marr, L.C. (2012). Mechanisms by which ambient humidity may affect viruses in aerosols. Appl. Environ. Microbiol. 78, 6781-6788. https://doi.org/10.1128/AEM.01658-12

Zhang, T. (2011). Study on Surface Tension and Evaporation Rate of Human Saliva, Saline, and Water Droplets (MS). West Virginia University Libraries. https://doi.org/10.33915/etd.2271

Zhao, H., Wu, Z., Li, W., Xu, J., Liu, H. (2019a). Interaction of two drops in the bag breakup regime by a continuous air jet. Fuel 236, 843-850. https://doi.org/10.1016/j.fuel.2018.09.067

Zhao, W., Zhang, Y., Xu, B. (2019b). An improved pseudopotential multi-relaxation-time lattice Boltzmann model for binary droplet collision with large density ratio. Fluid Dyn. Res. 51, 025510. https://doi.org/10.1088/1873-7005/aae443

Zhu, N., Zhang, D., Wang, W., Li, X., Yang, B., Song, J., Zhao, X., Huang, B., Shi, W., Lu, R., Niu, P., Zhan, F., Ma, X., Wang, D., Xu, W., Wu, G., Gao, G.F., Tan, W. (2020). A novel coronavirus from patients with pneumonia in China, 2019. N. Engl. J. Med. 382, 727-733. https://doi.org/10.105 6/NEJMoa2001017 